\title{
Integrated analysis of microRNA and mRNA expression and association with HIF binding reveals the complexity of microRNA expression regulation under hypoxia
}

Carme Camps $^{1 *}$, Harpreet K Saini ${ }^{2}$, David R Mole ${ }^{3}$, Hani Choudhry ${ }^{1,6}$, Martin Reczko ${ }^{4}$, José Afonso Guerra-Assunção ${ }^{2}$, Ya-Min Tian ${ }^{3}$, Francesca M Buffa ${ }^{5}$, Adrian L Harris ${ }^{5}$, Artemis G Hatzigeorgiou ${ }^{4}$, Anton J Enright ${ }^{2}$ and Jiannis Ragoussis ${ }^{1,4,7^{*}}$

\begin{abstract}
Background: In mammalians, HIF is a master regulator of hypoxia gene expression through direct binding to DNA, while its role in microRNA expression regulation, critical in the hypoxia response, is not elucidated genome wide. Our aim is to investigate in depth the regulation of microRNA expression by hypoxia in the breast cancer cell line MCF-7, establish the relationship between microRNA expression and HIF binding sites, pri-miRNA transcription and microRNA processing gene expression.
\end{abstract}

Methods: MCF-7 cells were incubated at 1\% Oxygen for 16, 32 and 48 h. SiRNA against HIF-1a and HIF-2a were performed as previously published. MicroRNA and mRNA expression were assessed using microRNA microarrays, small RNA sequencing, gene expression microarrays and Real time PCR. The Kraken pipeline was applied for microRNA-seq analysis along with Bioconductor packages. Microarray data was analysed using Limma (Bioconductor), ChIP-seq data were analysed using Gene Set Enrichment Analysis and multiple testing correction applied in all analyses.

Results: Hypoxia time course microRNA sequencing data analysis identified 41 microRNAs significantly up- and 28 down-regulated, including hsa-miR-4521, hsa-miR-145-3p and hsa-miR-222-5p reported in conjunction with hypoxia for the first time. Integration of HIF-1a and HIF-2a ChIP-seq data with expression data showed overall association between binding sites and microRNA up-regulation, with hsa-miR-210-3p and microRNAs of miR-27a/23a/24-2 and miR-30b/30d clusters as predominant examples. Moreover the expression of hsa-miR-27a-3p and hsa-miR-24-3p was found positively associated to a hypoxia gene signature in breast cancer. Gene expression analysis showed no full coordination between pri-miRNA and microRNA expression, pointing towards additional levels of regulation. Several transcripts involved in microRNA processing were found regulated by hypoxia, of which DICER (down-regulated) and AGO4 (up-regulated) were HIF dependent. DICER expression was found inversely correlated to hypoxia in breast cancer.

(Continued on next page)

\footnotetext{
*Correspondence: ccamps@well.ox.ac.uk; ioannis.ragoussis@mcgill.ca 'The Wellcome Trust Centre for Human Genetics, University of Oxford, Roosevelt Drive, Oxford, United Kingdom

${ }^{4}$ Institute of Molecular Oncology, Biomedical Sciences Research Center "Alexander Fleming", 34 Fleming Street, Vari 16672, Greece

Full list of author information is available at the end of the article
}

C Biomed Central

(c) 2014 Camps et al.; licensee BioMed Central Ltd. This is an Open Access article distributed under the terms of the Creative Commons Attribution License (http://creativecommons.org/licenses/by/2.0), which permits unrestricted use, distribution, and reproduction in any medium, provided the original work is properly cited. The Creative Commons Public Domain Dedication waiver (http://creativecommons.org/publicdomain/zero/1.0/) applies to the data made available in this article, unless otherwise stated. 
(Continued from previous page)

Conclusions: Integrated analysis of microRNA, mRNA and ChIP-seq data in a model cell line supports the hypothesis that microRNA expression under hypoxia is regulated at transcriptional and post-transcriptional level, with the presence of HIF binding sites at microRNA genomic loci associated with up-regulation. The identification of hypoxia and HIF regulated microRNAs relevant for breast cancer is important for our understanding of disease development and design of therapeutic interventions.

Keywords: MicroRNA, Hypoxia, HIF, Transcription factor, Gene regulation

\section{Background}

Hypoxia plays an important role in cancer, where the oxygen supply can be affected by tumour growth and subsequent alteration of the blood vessel network, leading to fatal consequences such as cell resistance to certain drugs and metastasis (reviewed in [1]). Under low cellular oxygen levels, the hypoxic inducible family of transcription factors (HIF) drive a complex transcriptional response that affects several biological processes relevant for the fate of the cells under these conditions, including glycolysis, angiogenesis and apoptosis [2]. HIF transcription factors are heterodimers composed of an alpha and a beta subunit. There are three HIF-alpha subunits, of which HIF- $1 \alpha$ and HIF- $2 \alpha$ have been shown to have important regulatory roles in the hypoxia response. HIFalpha protein levels are regulated by the Von Hippel Lindau (VHL) protein which mediates the ubiquitination of HIF-alpha by specifically recognising and binding to two prolyl-hydroxylated residues, resulting on a rapid elimination of HIF-alpha. HIF-alpha prolyl hydroxylation is catalysed by three homologous 2-oxoglutarate dependent dioxygenases, PHD1, PHD2 and PHD3. Further control of HIF-alpha expression is performed by another dioxygenase: Factor Inhibiting HIF 1 (FIH-1). FIH-1 catalyses the formation of a specific hydroxyasparaginyl residue in HIF-alpha that reduces its binding to the transcriptional coactivator p300 (for a review see $[3,4])$. Since the hydroxylation of HIF-alpha residues by PHDs and FIH is oxygen dependent, HIF-alpha is able to escape VHL recognition and further proteasomal degradation under hypoxic conditions, bind to p300 and HIF-beta and induce transcriptional changes that also affect microRNAs.

MicroRNAs are small noncoding RNA oligonucleotides ( 22 nucleotides) that regulate gene expression at post-transcriptional level. Their genomic organisation is heterogeneous. For instance approximately half of the microRNAs identified in mammals are found in intergenic regions whereas the other half are encoded within the open reading frame of other genes $[5,6]$. Nevertheless some microRNAs belonging to this last group have their own promoter. In addition microRNAs can be transcribed alone or in polycistronic units [7]. Independent of their origin, all microRNA transcripts undergo a complex process of biogenesis which takes part in different cellular compartments and involves many proteins. Briefly, first a microRNA containing transcript (pri-miRNA) is cleaved in the nucleus by a complex lead by DROSHA and DGCR8, generating 70-nucleotide long RNA molecules known as precursor microRNAs (pre-miRNAs). Pre-miRNAs are transported by EXPORTIN 5 from the nucleus to the cytoplasm where they are cleaved by DICER/TRBP complex, producing a 22 nucleotide microRNA duplex. This microRNA duplex is then loaded into the RNA-induced silencing complex (RISC) where ARGONAUTE (AGO) proteins have a key role on the recruitment of microRNAs and their mRNA targets. Finally one strand from the duplex mediates the regulation of target mRNAs by impairing their translation and/or by promoting their degradation. In most cases, there is a preference for the selection of one of the strands of the microRNA duplex in the RISC complex since both strands are detected at very assymetric levels, (for a review see [8]). Most of the earlier studies have focused on the most abundant strand (usually 5p), whereas the less abundant strand (also known as miRNA*) is considered to be degraded. However evidence is growing that miRNA* can actually be functional by regulating specific target mRNA $[9,10]$. Indeed, a mature miRNA is now designated with the suffix "-3p" or " $-5 \mathrm{p}$ " depending on the originating strand and we have used the same nomenclature in this work.

Several studies have been performed in cancer cell lines, endothelial cell lines and primary human cytotrophoblasts that have identified microRNAs regulated by hypoxia [11-20]. Most of these studies were done using microarray or Real time PCR (qPCR) based platforms, thus limiting the findings to a set of microRNAs. The overlap between the microRNAs found significantly regulated in these studies is low, probably due to the high variability generated by the fact of using different cell types, different experimental conditions and different technologies for measuring microRNA expression. The role of HIF in the regulation of microRNA expression has not been deeply investigated and only for a few microRNAs there is some experimental evidence that HIF is directly involved in the regulation of their expression under hypoxia [16,21-23]. Also some elements of 
the microRNA processing pathway have been recently reported to be regulated under hypoxia [14,18,24], but the direct involvement of HIF through DNA binding remains mostly unknown. The first screenings for hypoxia regulated microRNAs in breast cancer and other solid tumours, suggested that hypoxia could be a key factor in microRNA modulation in cancer [16]. Moreover, some hypoxia regulated microRNAs have been shown to have functional roles that are of great relevance in cancer. Therefore, it is important to shed more light in this area.

Our aim is to investigate the regulation of microRNA expression by hypoxia, with a special focus in the role of HIF in this process. We have chosen the breast cancer cell line MCF-7 as a system because we have already shown that the HIF- $1 \alpha$ and HIF- $2 \alpha$ proteins are present and stabilized across different hypoxia time points, starting at $16 \mathrm{~h}$, and we have characterised the role of both HIF- $1 \alpha$ and HIF- $2 \alpha$ in the regulation of gene expression in this system $[25,26]$. We have performed a comprehensive study, by integrating microRNA expression, gene expression and HIF- $1 \alpha$ and HIF- $2 \alpha$ chromatin immunoprecipitation (ChIP) data from the same breast cancer cell line MCF-7. We have used the latest next generation sequencing technology as well as established microarrays to generate microRNA expression profiles from a three point time course in hypoxia (16 h, $32 \mathrm{~h}$ and $48 \mathrm{~h}$ ). We present here microRNAs that are found consistently regulated at different hypoxia time points, including novel hypoxia related microRNAs. Moreover, we have used gene expression profiles to assess the relationship between microRNA and host gene for the microRNAs located in intragenic regions and HIF- $1 \alpha$ and HIF- $2 \alpha$ ChIP-seq data to identify binding sites close to microRNAs. Finally we also used these data sets to investigate the expression of the genes involved in the microRNA processing pathway and the involvement of HIF in their regulation.

\section{Results \\ Profiling the population of microRNAs in hypoxic MCF-7 cells by next-generation sequencing}

The total number of sequencing reads obtained was in the range between 32 and 35 million reads per sample. After filtering, sequencing reads with a length between 18 and 26 nucleotides represented $75.2-87.5 \%$ of total number of reads obtained initially (Additional file 1: Table S1). On average, 99\% of these reads (98.9-99.5\%) were mapped to the human genome using Bowtie, from which $75-85.9 \%$ had a unique mapping position (Additional file 1: Table S1). Most of the reads mapped to annotated regions (98-98.9\%), and as expected the vast majority of them matched genomic regions allocating microRNAs (94.5-96.1\% of reads mapped to annotated regions). The residual fraction of mapped reads not corresponding to microRNAs was distributed among a miscellanea of annotated regions including other RNA and small RNAs species, protein coding exons, pseudogenes and repeat molecules (Additional file 1: Table S1).

We were able to identify 502 microRNAs consistently across all conditions in MCF-7 cells (Additional file 1: Table S2). Among them, 412 microRNAs had strand $(-5 p /-3 p)$ annotations with $53.2 \%$ of $5 p$ and $46.8 \%$ of 3p-microRNAs. We found that 184 microRNAs were from only one strand (either-5p or-3p), so there were no detectable traces of the other hairpin arm and 114 microRNAs have both the $5 \mathrm{p}$ and $3 \mathrm{p}$ strands expressed.

The sequencing data also allowed us to identify precursors on different genomic loci that generate the same mature microRNA. For six mature microRNAs (hsa-let7f-5p, hsa-miR-16-5p, hsa-miR-181a-5p, hsa-miR-24-3p, hsa-miR-29b-3p and hsa-miR-30c-5p) we were able to identify microRNAs from the other strand derived from the two different precursors. These findings provide evidence that both loci of these particular microRNAs would be expressed in MCF-7 cells. Compared to a similar sequencing based study performed using human umbilical vein endothelial cells (HUVEC) [17], there was an overlap of 80 microRNA sequences.

\section{Differential expression of microRNAs under hypoxia in MCF-7 cells}

When looking at differential expression between hypoxia and normoxia, we found that the number of differentially expressed microRNAs increased along the time of exposure to hypoxia. We identified 1, 49 and 69 microRNAs up-regulated at $16 \mathrm{~h}, 32 \mathrm{~h}$ and $48 \mathrm{~h}$ of hypoxia respectively (adj.p-val $\leq 0.05$ ) whereas 4,37 and 50 microRNAs were detected as down-regulated at $16 \mathrm{~h}$, $32 \mathrm{~h}$ and $48 \mathrm{~h}$ of hypoxia respectively (adj.p-val $\leq 0.05$ ) (see Figure 1A). Among each group of significant microRNAs we found microRNAs from both $5 \mathrm{p}$ and $3 \mathrm{p}$ strands.

All microRNAs significantly changing in expression levels after $16 \mathrm{~h}$ of hypoxia remained altered in the same direction across the time course: the up-regulated hsamiR-210-3p and the down-regulated hsa-miR-4521, hsamiR-145-3p and hsa-miR-222-5p were also significantly up- and down-regulated, respectively, after $32 \mathrm{~h}$ and $48 \mathrm{~h}$ of hypoxia whereas the down-regulated hsa-miR-29b-1-5p remained significantly decreased after $32 \mathrm{~h}$ of hypoxia but not $48 \mathrm{~h}$ (Figure $1 \mathrm{~A}$ and Tables 1 and 2). The number of significantly up- or down-regulated microRNAs increases substantially at 32 and 48 h of hypoxia, with a considerable overlap between these time points: 40 and 24 microRNAs were found to be significantly up-regulated and down-regulated, respectively, at both hypoxia time points (Figure 1A and Tables 1 and 2, adj.p-val <0.05). The induction of HIF protein levels across the hypoxia time course was monitored by immunoblotting, showing 
A)

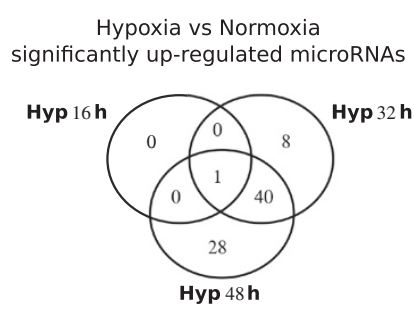

B)

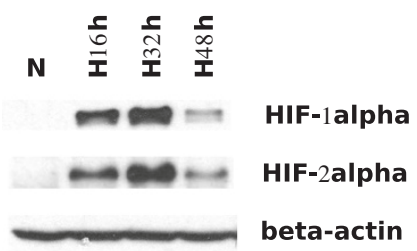

C)

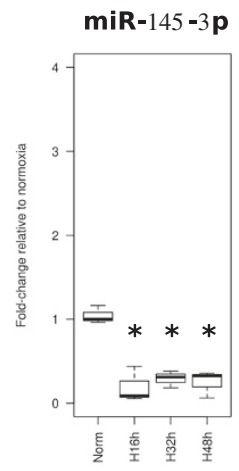

miR-145 -5p

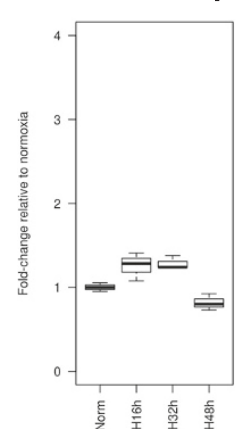

Hypoxia vs Normoxia

significantly down-regulated microRNAs

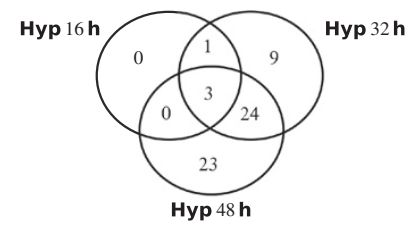

\section{beta-actin}
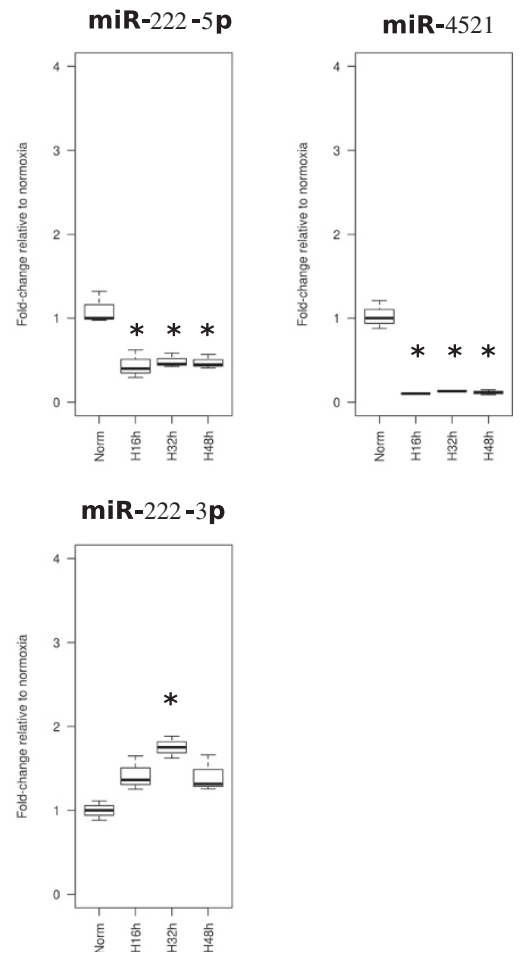

Figure 1 Hypoxia regulated microRNAs in MCF-7 cells. microRNA sequencing data generated from a hypoxia time course in MCF-7 (16, 32 and $48 \mathrm{~h}$ at $1 \%$ Oxygen) was analysed and the overlap between the microRNAs found significantly up- or down-regulated at each time point compared to normoxia control (adj.p-val <0.05) has been represented in Venn diagrams (A). The levels of HIF-1a and HIF-2a were measured by immunoblotting across the hypoxia time course ( $\mathrm{H} 16 \mathrm{~h}, \mathrm{H} 32 \mathrm{~h}, \mathrm{H} 48 \mathrm{~h})$ and normoxia (N), with beta-actin used as a control (B). The down-regulation of miR-4521, miR-145-3p and miR-222-5p in hypoxia was validated by qPCR (C). Fold-changes (in linear scale) obtained for each microRNA at each time point relative to normoxia control are represented in boxplots ( ${ }^{*}$ significant fold-change compared to normoxia after ANOVA followed by pairwise t-test, adj.p-val $\leq 0.05$ ). The expression of miR-145-5p and miR-222-3p was also assessed by qPCR, confirming a different pattern of expression as compared to their counterpart strands.

that both HIF- $1 \alpha$ and HIF- $2 \alpha$ are induced at $16 \mathrm{~h}$, increase at $32 \mathrm{~h}$ of hypoxia and drop at $48 \mathrm{~h}$ (see Figure 1B).

We have previously demonstrated the up-regulation of hsa-miR-210-3p (previously annotated as hsa-miR-210) across a hypoxia time course in MCF-7 cells [27], but this in depth study has revealed a number of hypoxia regulated
microRNAs that have not been reported in previous studies [11-20] (highlighted in bold in Tables 1 and 2).

Agilent microRNA microarrays (3 biological replicates per condition) were also used to complement and validate the sequencing data in a broad manner, as the two technologies have their own strengths and weaknesses in 
Table 1 MicroRNAs found significantly up-regulated in MCF7 cells exposed to hypoxia by analysis of microRNA-seq data

\begin{tabular}{|c|c|c|c|c|c|c|}
\hline \multicolumn{7}{|c|}{ MicroRNAs significantly up-regulated in hypoxia at 16,32 and $48 \mathrm{~h}$ (adj.p-val $\leq 0.05$ ) } \\
\hline microRNAs & FC H16h & adj.p-val & FC H32h & adj.p-val & FC H48h & adj.p-val \\
\hline hsa-miR-210-3p & 3.24 & 0.0001 & 4.03 & $5.46 \mathrm{e}-07$ & 3.96 & $8.41 \mathrm{e}-07$ \\
\hline \multicolumn{7}{|c|}{ MicroRNAs significantly up-regulated in hypoxia at 32 and $48 \mathrm{~h}$ (adj.p-val $\leq 0.05$ ) } \\
\hline microRNAs & FC H16h & adj.p-val & FC H32h & adj.p-val & FC H48h & adj.p-val \\
\hline hsa-miR-1 & 1.86 & 0.4806 & 2.25 & 0.026223 & 3.12 & 0.000682 \\
\hline hsa-miR-106b-3p & 1.10 & 0.9629 & 2.15 & 0.029336 & 1.94 & 0.048003 \\
\hline hsa-miR-1246 & 1.53 & 0.9046 & 5.08 & 0.000085 & 3.87 & 0.001346 \\
\hline hsa-miR-1269a & 1.18 & 0.9399 & 2.43 & 0.005459 & 1.94 & 0.033244 \\
\hline hsa-miR-140-3p & 1.11 & 0.9629 & 2.21 & 0.016949 & 2.01 & 0.027275 \\
\hline hsa-miR-141-5p & 1.26 & 0.9046 & 1.78 & 0.049237 & 1.93 & 0.018787 \\
\hline hsa-miR-143-3p & 1.82 & 0.2553 & 2.80 & 0.000136 & 1.90 & 0.021242 \\
\hline hsa-miR-151a-3p & 1.35 & 0.9046 & 2.47 & 0.014476 & 2.34 & 0.016879 \\
\hline hsa-miR-181c-3p & 1.12 & 0.9629 & 1.89 & 0.038509 & 1.84 & 0.035990 \\
\hline hsa-miR-192-5p & 1.33 & 0.8665 & 2.14 & 0.009894 & 2.24 & 0.003741 \\
\hline hsa-miR-194-5p & 1.09 & 0.9629 & 1.96 & 0.042635 & 2.28 & 0.008532 \\
\hline hsa-miR-195-3p & 1.18 & 0.9555 & 2.49 & 0.007142 & 2.29 & 0.012846 \\
\hline hsa-miR-203a & 1.21 & 0.9242 & 1.93 & 0.039759 & 1.93 & 0.030635 \\
\hline hsa-miR-215-5p & 1.28 & 0.9046 & 2.92 & 0.001669 & 2.23 & 0.017671 \\
\hline hsa-miR-27a-5p & 1.22 & 0.9046 & 1.90 & 0.026536 & 2.19 & 0.003759 \\
\hline hsa-miR-28-3p & 0.97 & 0.9774 & 2.15 & 0.021442 & 2.04 & 0.025454 \\
\hline hsa-miR-3065-3p & 1.27 & 0.9046 & 2.13 & 0.017787 & 2.67 & 0.000890 \\
\hline hsa-miR-30d-5p & 1.24 & 0.9046 & 2.38 & 0.005459 & 3.31 & 0.000050 \\
\hline hsa-miR-30d-3p & 1.43 & 0.8010 & 2.04 & 0.018042 & 1.80 & 0.041199 \\
\hline hsa-miR-30e-3p & 1.26 & 0.9046 & 2.00 & 0.033309 & 2.35 & 0.005147 \\
\hline hsa-miR-3140-3p & 1.13 & 0.9629 & 2.17 & 0.049237 & 2.31 & 0.025454 \\
\hline hsa-miR-3158-3p & 2.19 & 0.0594 & 3.29 & 0.000032 & 2.65 & 0.000730 \\
\hline hsa-miR-338-5p & 1.15 & 0.9604 & 2.11 & 0.018185 & 2.72 & 0.000672 \\
\hline hsa-miR-33b-5p & 1.27 & 0.9046 & 2.87 & 0.000509 & 1.97 & 0.025454 \\
\hline hsa-miR-203b-3p & 1.18 & 0.9555 & 2.11 & 0.047389 & 2.26 & 0.023495 \\
\hline hsa-miR-3619-3p & 1.30 & 0.9242 & 3.11 & 0.004682 & 4.15 & 0.000159 \\
\hline hsa-miR-3677-3p & 1.77 & 0.6186 & 2.94 & 0.002596 & 2.73 & 0.003759 \\
\hline hsa-miR-378c & 1.02 & 0.9835 & 2.11 & 0.020104 & 1.88 & 0.040362 \\
\hline hsa-miR-378d & 1.04 & 0.9629 & 2.39 & 0.013088 & 2.08 & 0.029016 \\
\hline hsa-miR-378i & 1.38 & 0.8770 & 2.63 & 0.003952 & 2.69 & 0.002146 \\
\hline hsa-miR-3913-5p & 1.06 & 0.9629 & 1.91 & 0.041667 & 2.26 & 0.006343 \\
\hline hsa-miR-3928-3p & 1.22 & 0.9250 & 2.28 & 0.023169 & 2.12 & 0.030094 \\
\hline hsa-miR-4504 & 2.14 & 0.4257 & 2.97 & 0.006637 & 2.55 & 0.019065 \\
\hline hsa-miR-4746-5p & 1.37 & 0.8665 & 2.13 & 0.018185 & 2.17 & 0.011517 \\
\hline hsa-miR-4760-5p & 1.82 & 0.2984 & 2.04 & 0.017700 & 1.80 & 0.040620 \\
\hline hsa-miR-548a-3p & 1.12 & 0.9629 & 2.55 & 0.032600 & 2.33 & 0.046620 \\
\hline hsa-miR-627-5p & 1.44 & 0.8474 & 2.72 & 0.002857 & 2.29 & 0.011721 \\
\hline hsa-miR-92b-3p & 1.19 & 0.9242 & 1.95 & 0.032600 & 2.22 & 0.006343 \\
\hline hsa-miR-942-5p & 1.25 & 0.9046 & 2.08 & 0.016836 & 2.16 & 0.007682 \\
\hline hsa-miR-99b-5p & 1.15 & 0.9629 & 2.39 & 0.018617 & 3.09 & 0.001097 \\
\hline
\end{tabular}


Table 1 MicroRNAs found significantly up-regulated in MCF7 cells exposed to hypoxia by analysis of microRNA-seq data (Continued)

\begin{tabular}{|c|c|c|c|c|c|c|}
\hline \multicolumn{7}{|c|}{ MicroRNAs significantly up-regulated in hypoxia by $\mathrm{QPCR}^{\dagger}$} \\
\hline microRNAs & FC H16h & adj.p-val & $\mathrm{FC} \mathrm{H} 32 \mathrm{~h}$ & adj.p-val & FC H48h & adj.p-val \\
\hline hsa-miR-24-2-5p & 1.08 & $\geq 0.05$ & 1.65 & $\leq 0.05$ & 1.81 & $\leq 0.05$ \\
\hline hsa-miR-27a-3p & 1.93 & $\leq 0.05$ & 2.84 & $\leq 0.05$ & 2.02 & $\leq 0.05$ \\
\hline hsa-miR-30b-3p & 1.58 & $\geq 0.05$ & 2.10 & $\leq 0.05$ & 2.40 & $\leq 0.05$ \\
\hline hsa-miR-30b-5p & 1.20 & $\geq 0.05$ & 2.23 & $\leq 0.05$ & 1.81 & $\leq 0.05$ \\
\hline
\end{tabular}

MicroRNAs in each section of the table are arranged in numerical order for ease of reading. Fold-change in linear scale (FC) and adjusted $p$ value (adj.p-val) are shown for each microRNA at each hypoxia time. MicroRNAs which regulation by hypoxia has not been reported before are highlighted in bold. Last section shows microRNAs for which sequencing data did not produce significant results but were validated as up-regulated by qPCR $\left({ }^{\dagger}\right)$.

Hsa-miR-27a-3p was also found significantly up-regulated across the hypoxia time course in Agilent microarray data.

microRNA profiling [28]. We found 228 microRNAs commonly detected between sequencing and microarrays (Additional file 1: Table S3), with a significant correlation between the number of reads and array intensity (Spearman: 0.57 (average), $\mathrm{p}$-val $\leq 0.05$ ). When comparing the fold changes obtained for each microRNA across the time course in each platform there is overall a significant correlation (Pearson: 0.30 (average), p-val $\leq 0.05$ ). Most of the compared microRNAs show a clear trend towards the same direction in both platforms despite some punctual disagreements (see Additional file 2: Figure S1). Moreover around half of the microRNAs found significantly regulated through next-generation sequencing are also found significantly regulated in the microarray experiment (Additional file 2: Figure S1 and Additional file 1: Table S3).

In order to further validate the sequencing results obtained here, we performed qPCR to test the expression levels of hsa-miR-4521, hsa-miR-145-3p and hsamiR-222-5p. Indeed, all microRNAs were validated as down-regulated in agreement with the sequencing data (Figure 1C, adj.p-val $\leq 0.05$ ). The expression levels of hsa-miR-145-5p and hsa-miR-222-3p were found either not changed or slightly up by qPCR (Figure $1 C$ ). This suggests that one strand for these microRNAs is specifically down-regulated under hypoxic conditions and may have been functional as compared to the other strand.

\section{Role of HIF in microRNA regulation under hypoxia}

Direct regulation of microRNA expression by HIF- $1 \alpha$ and HIF- $2 \alpha$ in MCF-7 was assessed by ChIP-seq, while stabilization of the two proteins in the same cells under hypoxia has been published previously $[25,26]$. We used the 500 published high-stringency HIF binding sites [29] in order to look for microRNAs that map to within $50 \mathrm{~kb}$ of each binding site. Gene Set Enrichment Analysis (GSEA) against the fold change by hypoxia at each of the three time points shows a weak enrichment for HIFbinding microRNAs amongst those up-regulated by hypoxia, which becomes more apparent at the longer time points (Figure 2A-C). The heat map (Figure 2D) shows the fold change at each time point for each microRNA locus containing a HIF binding site. The most upregulated microRNA containing a HIF-binding site is indeed hsa-miR-210-3p, but there are clearly other microRNAs (Additional file 1: Table S4). In Figure 2E and F we show the tracks for two loci of particular interest, MIR30D and MIR27A, which are near the top of the list. Indeed, hsa-miR-30d-5p, hsa-miR-30d-3p and hsa-miR-27a-5p are significantly up-regulated at $32 \mathrm{~h}$ and $48 \mathrm{~h}$ of hypoxia according to our sequencing data. Both microRNA loci have un-annotated, hypoxia inducible host transcripts that appear in directional ribo-depleted RNA-seq data produced by exposing the MCF-7 cells to hypoxia (24 h $1 \%$ hypoxia) [30] and strong HIF-binding ChIP-seq peaks (Figure 2E and F).

Hsa-miR-27a is within a close distance to other two microRNAs, hsa-miR-23a and hsa-miR-24-2, whereas hsa-miR-30d is also very close to hsa-miR-30b. They are reported to be clustered microRNAs that seem to be transcribed in the same pri-miRNA, which agrees with our previous observations concerning the host genes for these microRNAs. Therefore, we checked the expression of these microRNAs by qPCR, validating the up-regulations seen in sequencing results for hsa-miR-30d-5p, hsamiR-30d-3p and hsa-miR-27a-5p (Figure $3 \mathrm{~A}$ and B, adj.p-val $\leq 0.05$ ). In addition we found hsa-miR-27a-3p significantly up-regulated across the whole hypoxia time course, hsa-miR-23a-3p up-regulated at $48 \mathrm{~h}$ and hsamiR-24-2-5p, hsa-miR-30b-5p and hsa-miR-30b-3p significantly up-regulated at 32 and $48 \mathrm{~h}$ (Figure $3 \mathrm{~A}$ and $B$, adj-p-val $\leq 0.05$ ). It is worth to mention that hsamiR-27a-3p was also found significantly up-regulated across all hypoxia time course in Agilent microarray data (Additional file 1: Table S3). All these results would further support the role of HIF on regulating the expression of these microRNA clusters under hypoxic conditions. The results link genome wide HIF occupancy under hypoxic conditions to the up-regulation of pri-miRNAs and mature microRNAs. 
Table 2 MicroRNAs found significantly down-regulated in MCF7 cells exposed to hypoxia by analysis of microRNA-seq data

\begin{tabular}{|c|c|c|c|c|c|c|}
\hline \multicolumn{7}{|c|}{ MicroRNAs significantly down-regulated in hypoxia at 16,32 and $48 \mathrm{~h}$ (adj.p-val $\leq 0.05$ ) } \\
\hline miRNAs & FC H16h & adj.p-val & FC H32h & adj.p-val & FC H48h & adj.p-val \\
\hline hsa-miR-145-3p & 0.16 & $5.60 \mathrm{E}-06$ & 0.13 & $1.23 \mathrm{E}-07$ & 0.10 & $1.16 \mathrm{E}-08$ \\
\hline hsa-miR-222-5p & 0.41 & 0.02943717 & 0.34 & 0.000541974 & 0.30 & $7.58 \mathrm{E}-05$ \\
\hline hsa-miR-4521 & 0.16 & 2.29E-07 & 0.09 & $9.76 \mathrm{E}-12$ & 0.09 & $7.42 \mathrm{E}-12$ \\
\hline \multicolumn{7}{|c|}{ MicroRNAs significantly down-regulated in hypoxia at 16 and $32 \mathrm{~h}$ (adj.p-val $\leq 0.05$ ) } \\
\hline miRNAs & FC H16h & adj.p-val & FC H32h & adj.p-val & FC H48h & adj.p-val \\
\hline hsa-miR-29b-1-5p & 0.41 & 0.02943717 & 0.53 & 0.049237065 & 0.64 & 0.160459153 \\
\hline \multicolumn{7}{|c|}{ MicroRNAs significantly down-regulated in hypoxia at 32 and $48 \mathrm{~h}$ (adj.p-val $\leq 0.05$ ) } \\
\hline miRNAs & FC H16h & adj.p-val & FC H32h & adj.p-val & FC H48h & adj.p-val \\
\hline hsa-let-7f-1-3p & 0.58 & 0.618581404 & 0.37 & 0.003952372 & 0.46 & 0.02280333 \\
\hline hsa-miR-1260a & 0.59 & 0.676561681 & 0.25 & 0.000224356 & 0.45 & 0.027900682 \\
\hline hsa-miR-1260b & 0.52 & 0.480646057 & 0.19 & $1.21 \mathrm{E}-05$ & 0.38 & 0.008394683 \\
\hline hsa-miR-1275 & 0.98 & 0.983716794 & 0.46 & 0.038509311 & 0.25 & 0.000158578 \\
\hline hsa-miR-15b-3p & 0.92 & 0.962873516 & 0.32 & 0.003952372 & 0.36 & 0.007476629 \\
\hline hsa-miR-19a-3p & 1.59 & 0.866491946 & 0.37 & 0.036219879 & 0.37 & 0.025453723 \\
\hline hsa-miR-19b-3p & 1.55 & 0.866491946 & 0.33 & 0.018184509 & 0.34 & 0.016879484 \\
\hline hsa-miR-19b-1-5p & 0.53 & 0.472759683 & 0.33 & 0.002596151 & 0.31 & 0.000890148 \\
\hline hsa-miR-22-3p & 1.39 & 0.904640745 & 0.41 & 0.039758527 & 0.40 & 0.026856552 \\
\hline hsa-miR-221-5p & 0.82 & 0.904640745 & 0.54 & 0.041249481 & 0.36 & 0.000264743 \\
\hline hsa-miR-23a-5p & 0.35 & 0.225229269 & 0.30 & 0.016836038 & 0.16 & 0.000197073 \\
\hline hsa-miR-23b-5p & 0.46 & 0.059350069 & 0.43 & 0.003952372 & 0.43 & 0.002649597 \\
\hline hsa-miR-296-3p & 0.46 & 0.298355571 & 0.34 & 0.003952372 & 0.21 & $1.55 \mathrm{E}-05$ \\
\hline hsa-miR-32-3p & 0.86 & 0.955522425 & 0.48 & 0.036493987 & 0.33 & 0.000822847 \\
\hline hsa-miR-33a-3p & 0.44 & 0.618581404 & 0.22 & 0.012191307 & 0.17 & 0.003599729 \\
\hline hsa-miR-3613-5p & 0.88 & 0.962873516 & 0.36 & 0.002314 & 0.38 & 0.002622677 \\
\hline hsa-miR-424-3p & 0.64 & 0.720598475 & 0.31 & 0.000695205 & 0.23 & $9.65 \mathrm{E}-06$ \\
\hline hsa-miR-4466 & 0.53 & 0.50269118 & 0.13 & $1.23 \mathrm{E}-07$ & 0.19 & $1.72 \mathrm{E}-05$ \\
\hline hsa-miR-455-3p & 1.29 & 0.904640745 & 0.46 & 0.026536474 & 0.45 & 0.016879484 \\
\hline hsa-miR-505-5p & 0.73 & 0.866491946 & 0.36 & 0.001669409 & 0.35 & 0.000890148 \\
\hline hsa-miR-573 & 0.66 & 0.773087527 & 0.18 & $1.88 \mathrm{E}-07$ & 0.09 & $4.29 \mathrm{E}-12$ \\
\hline hsa-miR-92a-1-5p & 0.59 & 0.720598475 & 0.22 & 0.000120735 & 0.20 & $2.52 \mathrm{E}-05$ \\
\hline hsa-miR-93-3p & 0.63 & 0.720598475 & 0.42 & 0.016836038 & 0.37 & 0.003599729 \\
\hline hsa-miR-940 & 1.11 & 0.962873516 & 0.32 & 0.002314 & 0.27 & 0.000255512 \\
\hline
\end{tabular}

MicroRNAs in each section of the table are arranged in numerical order for ease of reading. Fold-change in linear scale (FC) and adjusted $p$ value (adj.p-val) are shown for each microRNA at each hypoxia time. MicroRNAs which regulation by hypoxia has not been reported before have been highlighted in bold.

Association of hypoxia regulated microRNAs with a hypoxia gene signature in breast cancer samples

Previously, we have reported the direct correlation of hsa-miR-210-3p with a hypoxia score based on the expression of 99 genes [31] in a series of breast cancer tumours [27]. Since microRNA expression profiles for this breast cancer series have been recently published (GSE22220) [32], we have investigated the correlation of the hypoxia score with microRNA expression in this series. Among the positively correlated microRNAs to hypoxia in breast cancer (Spearman rank test, adj.p-val $\leq 0.05$ ), there are two microRNAs that overlap with our list of hypoxia induced microRNAs: hsa-miR-210-3p and hsa-miR-27a-3p, which is derived from the MIR27A cluster (see Table 3). Interestingly another microRNA generated from the same cluster, hsa-miR-24-3p, is also directly correlated to hypoxia in breast cancer (Table 3). Although no regulation by hypoxia has been detected for this microRNA in our MCF-7 cells, this could be due to the fact that hsamiR-24-3p is encoded in two different genomic regions 


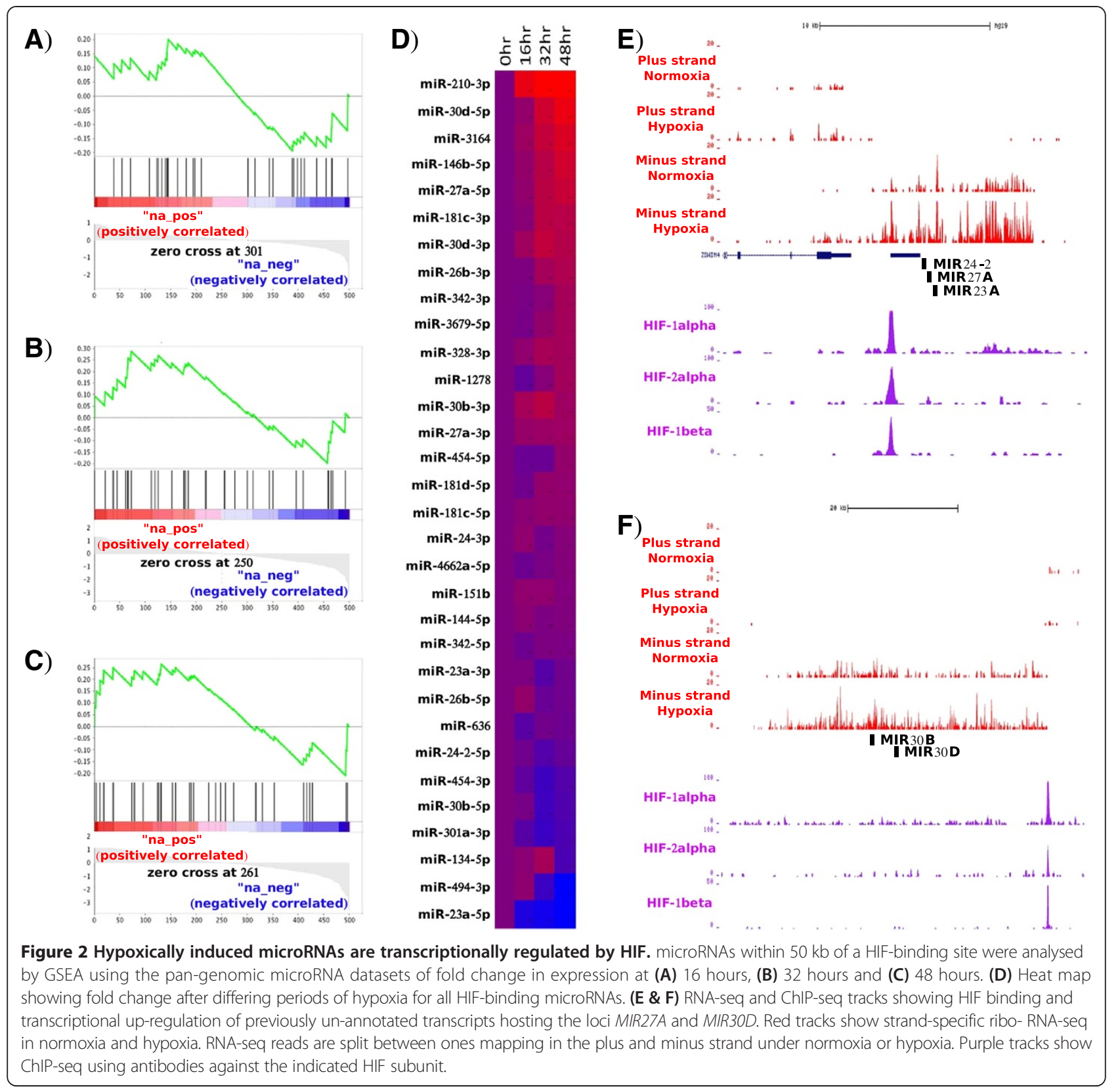

and therefore can be subjected to other regulatory mechanisms that contribute to its final levels. However it is important to highlight that three of the microRNAs for which we have identified a HIF binding site at a close distance appear to be directly correlated to hypoxia in breast cancer. This data demonstrates for the first time that hsa-miR-27a-3p and hsa-miR-24-3p are regulated by hypoxia in vivo in breast cancer and that this regulation is possibly dependent on HIF.

\section{microRNAs encoded within host genes}

Some of the microRNAs found up- or down-regulated under hypoxia are located within intronic regions of protein coding genes. Therefore, we generated mRNA expression profiles of the same time course samples using Illumina arrays. If only considering microRNAs with a unique genomic position, we found 185 microRNAs encoded within 120 host genes that were detected in our microarray experiment (Additional file 1: Table S5). No correlation was found between microRNA expression and host gene expression at any hypoxia time point (Spearman rank correlation: $0.137(\mathrm{P} . \mathrm{val}=0.0613$ ) at $16 \mathrm{~h} ;-0.0949(\mathrm{P} . \mathrm{val}=0.1961)$ at $32 \mathrm{~h} ;-0.0127(\mathrm{P} . v a l=0.8621)$ at $48 \mathrm{~h}$ ). Looking at the expression of genes containing microRNAs, we observed that they do not show a lot of variation. When dividing them in 3 groups according to 

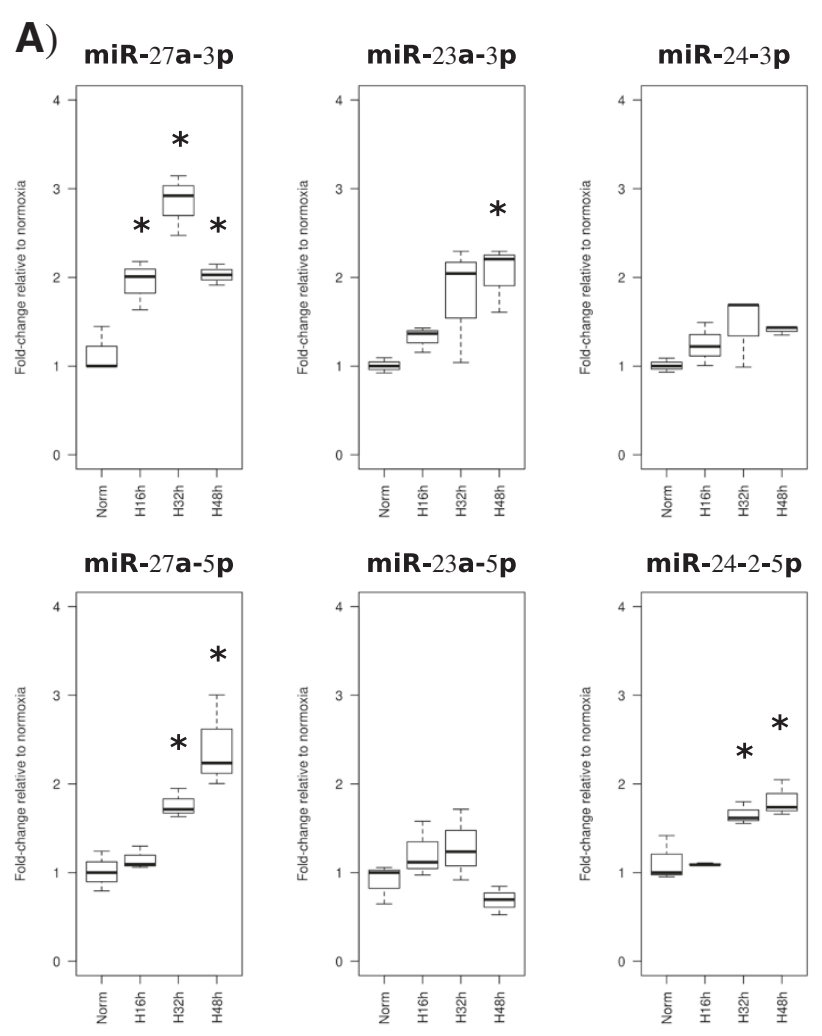

B)
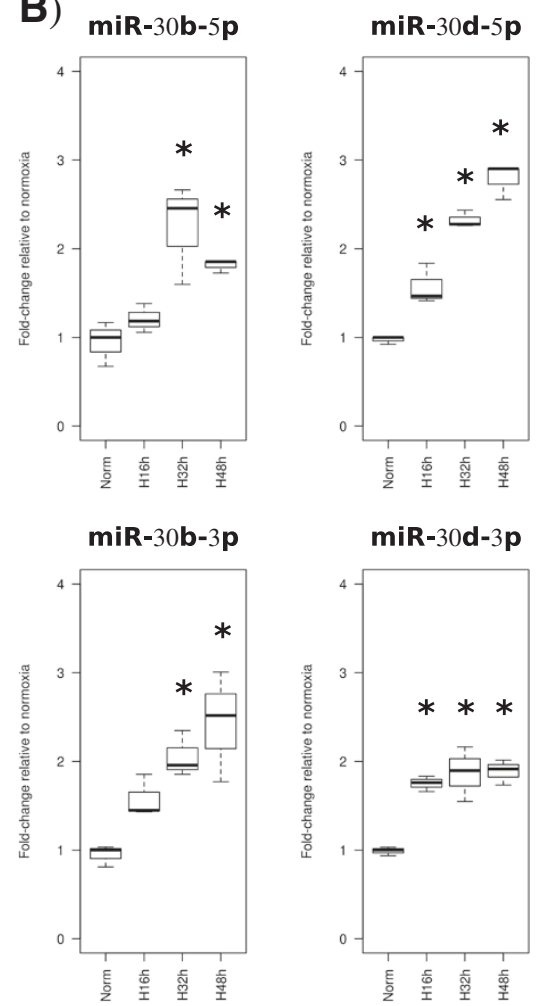

Figure 3 (See legend on next page.) 
(See figure on previous page.)

Figure 3 Hypoxic regulation of microRNAs clustered to MIR27A and MIR30D. QPCR was used to assess the expression of both $5 p$ and $3 p$ arms of two microRNA clusters that are within 50 kb of a HIF-binding site: hsa-miR-27a, hsa-miR-23a and hsa-miR-24-2 (A) and hsa-miR-30d and hsa-miR-30b (B). Fold-changes (in linear scale) obtained for each microRNA at each time point relative to normoxia control are represented in boxplots (*significant fold-change compared to normoxia after ANOVA followed by pairwise t-test, adj.p-val $\leq 0.05$ ).

whether they were significantly up-regulated, significantly down-regulated or without changes in hypoxia, the average fold-change of the up- or down-regulated groups is not very different to the group without changes (Figure 4A), suggesting that these genes are very lightly regulated by hypoxia at transcriptional level. For each of the group of genes, we represented boxplots with the expression of the microRNAs they contained (Figure 4B), showing the lack of correlation between the average fold-change of the microRNAs and the average fold-change of the corresponding host genes. Similarly from the microRNA point of view, we divided the microRNAs located within a host gene in three groups depending on their fold-change expression at $32 \mathrm{~h}$ and $48 \mathrm{~h}$ of hypoxia (up-regulated, down-regulated or no change, Figure 4C). Then for each group of microRNAs, we represented the fold-changes of the corresponding host genes (Figure 4D). While upregulated and down-regulated microRNAs showed an average fold-change of 2 and 0.5 respectively (Figure 4C), the average fold-change of the corresponding host genes was very similar and close to 1 for both groups (Figure 4D). The picture is the same when Agilent microarray data are used to determine microRNA expression fold changes instead of small RNA-seq (Additional file 3: Figure S2). Detailed examples of these relationships can be seen in Additional file 1: Table S6. In general we observed around a $50 \%$ agreement between microRNA and host gene expression. In order to gain more information, we focused on cases where there is no correlation between microRNA and host gene expression and performed additional validations. The first example is hsa-miR-942-5p, found significantly up-regulated at 32 and $48 \mathrm{~h}$, whereas its hosting gene TTF2 was significantly down-regulated in array data (Additional file 1: Tables S5 and S6). Indeed TTF2 was found significantly down-

Table 3 MicroRNAs found up-regulated in hypoxic MCF-7 and positively correlated to hypoxia in breast cancer

\begin{tabular}{llc}
\hline miRNAs & rho & adj.p-val \\
\hline hsa-miR-210-3p & 0.52 & $1.12 \mathrm{E}-15$ \\
hsa-miR-27a-3p & 0.31 & $5.03 \mathrm{E}-06$ \\
hsa-miR-24-3p $^{+}$ & 0.36 & $1.15 \mathrm{E}-07$
\end{tabular}

MicroRNAs are arranged according to significance to hypoxia signature in breast cancer. Correlation coefficient (rho) and adjusted $\mathrm{p}$ value (adj.p-val) are shown. ${ }^{\dagger}$ Not significantly regulated in hypoxia in MCF-7 cells. However it is part with hsa-miR-27a-3p of the same cluster, which has a HIF binding site mapped close. regulated in hypoxia by $\mathrm{qPCR}$ (adj.p-val $\leq 0.05$, data not shown). Also hsa-miR-3140-3p is up-regulated in sequencing data whereas the host gene $F B X W 7$ is down-regulated in array data (Additional file 1: Tables S5 and S6). FBXW7 has four isoforms and only two would contain MIR3140 within its sequence (Figure $5 \mathrm{~A}$ ). We designed a pair of primers (Figure 5A, F1 and R1) that would amplify three isoforms, including two that do not include the MIR3140 locus and a pair of primers specific for the two isoforms containing MIR3140 (Figure 5A, F2 and R2). All products were found significantly down-regulated in hypoxia by qPCR (Figure 5A, adj.p-val $\leq 0.05$ ).

Another interesting example is the cluster composed by hsa-miR-106b and hsa-miR-93 and their host gene MCM7. The microRNA sequencing data showed a significant up-regulation of hsa-miR-106b-3p and downregulation of hsa-miR-93-3p at $32 \mathrm{~h}$ and $48 \mathrm{~h}$ of hypoxia, whereas MCM7 was significantly down-regulated according to array data (Additional file 1: Tables S5 and S6). MCM7 has two isoforms and was confirmed as significantly downregulated in hypoxia by $\mathrm{qPCR}$ when primers able to amplify both isoforms were used (Figure 5B, primers F0 and R0). However when using specific primers for testing the expression of each isoform separately, we found one isoform significantly down-regulated at all hypoxia times (Figure 5B, primers F1 and R1, adj.p-val $\leq 0.05$ ) whereas the other isoform was significantly up-regulated at 32 and $48 \mathrm{~h}$ (Figure 5B, primers F2 and R2, adj.p-val $\leq 0.05$ ). This isoform specific regulation would match the regulation of the microRNAs provided they are processed from preferred isoforms.

\section{Changes in expression of microRNA processing genes due to hypoxia}

Overall there is a certain degree of coordination between host gene and microRNA expression under hypoxia but also discrepancy. This suggests that other factors may be involved in the changes observed in mature microRNA expression under hypoxia. We used the gene expression profiles to specifically monitor any change in the expression of genes coding for proteins involved in the microRNA maturation pathway in hypoxic MCF-7 cells. Indeed, significant changes (see Additional file 1: Table S7, adj.p-val < 0.05) were detected in the expression of several genes, including DDX5 (encoding P68 which forms part of the protein complex lead by DROSHA), XPO5 and RAN (encoding EXPORTIN5 and RAN, respectively, both 


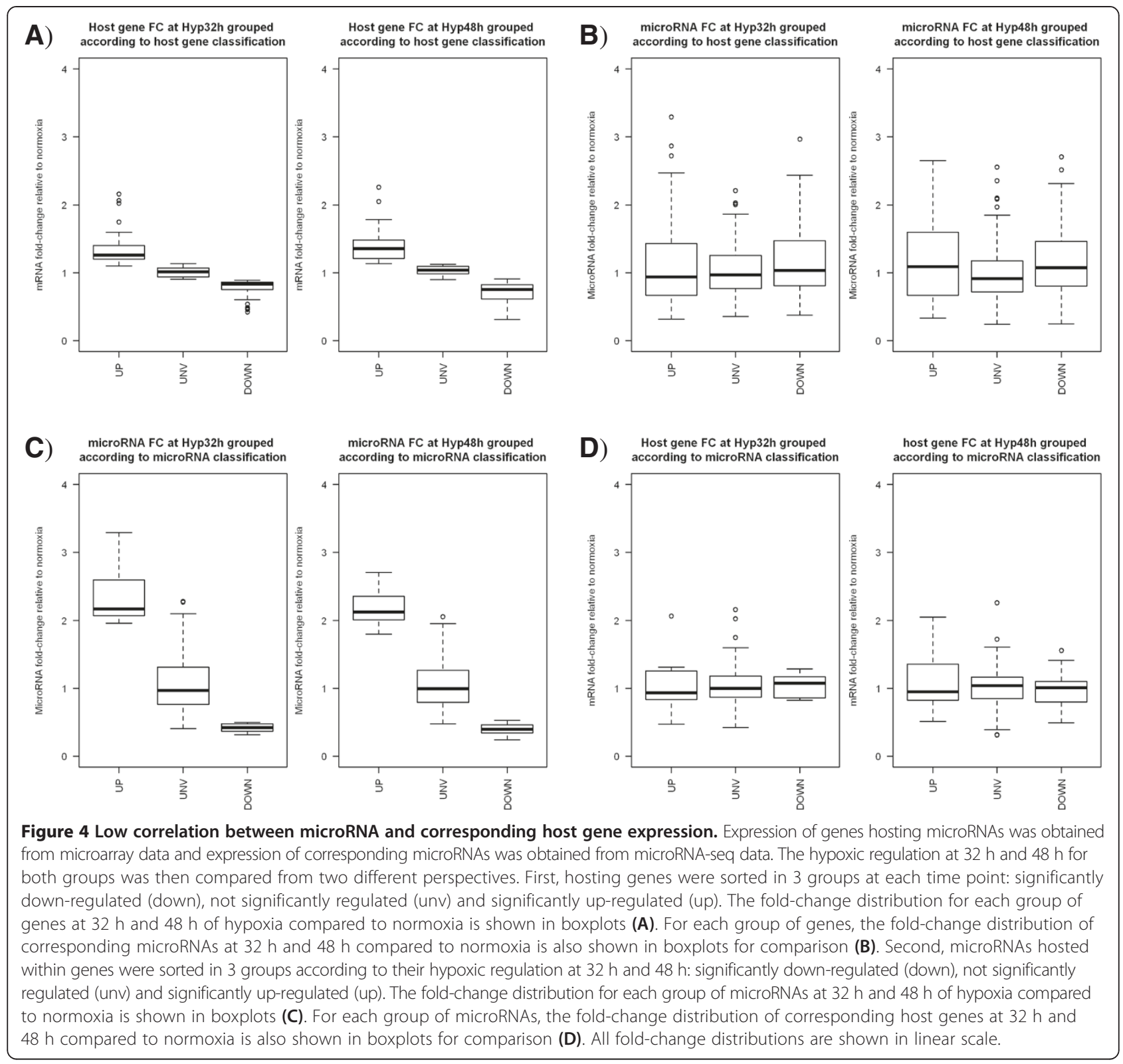

involved in microRNA transport from the nucleus to the cytoplasm), DICER and EIF2C2 and EIF2C4 (encoding $\mathrm{AGO} 2$ and $\mathrm{AGO} 4$, respectively, both associated to RISC complex). We also found that some of these changes were HIF- $1 \alpha$ and HIF- $2 \alpha$ dependent based on previous data, in which gene expression regulations dependent on these transcription factors were studied through microarray profiles after short interfering (si) RNA-based suppression of HIF subunits [26]. In order to validate these results, we performed qPCR using the MCF-7 hypoxia time course, and two HIF- $1 \alpha$ and HIF$2 \alpha$ siRNA sample sets (Figure 6). Indeed, we could confirm a significant down-regulation of DDX5, XPO5, RAN,
EIF2C2 and DICER consistently across the hypoxia time course in MCF-7 cells, as well as a significant upregulation of EIF2C4 (Figure 6). We also confirmed by qPCR a significant up-regulation of both genes DICER and EIF2C2 when HIF- $1 \alpha$ and HIF- $2 \alpha$ are silenced in MCF-7 compared to the control, in both independent sample sets (Figure 6). Concerning EIF2C4, its expression was found significantly down-regulated upon HIF- $1 \alpha$ silencing (Figure 6). Therefore the down-regulation of DICER and EIF2C2 under hypoxia is dependent on both HIF- $1 \alpha$ and HIF-2 $\alpha$, whereas the up-regulation of EIF2C4 seems to be only dependent on HIF- $1 \alpha$. Changes observed in DDX5, XPO5 and RAN in hypoxic MCF-7 seem to be 
A)
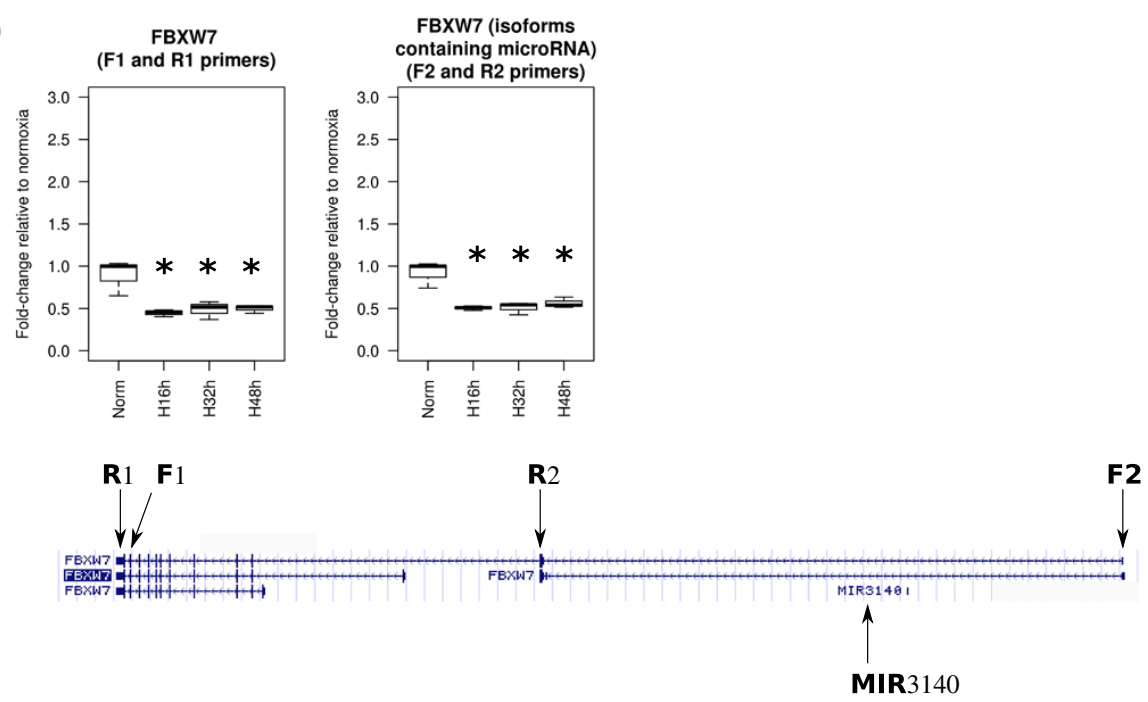

B)
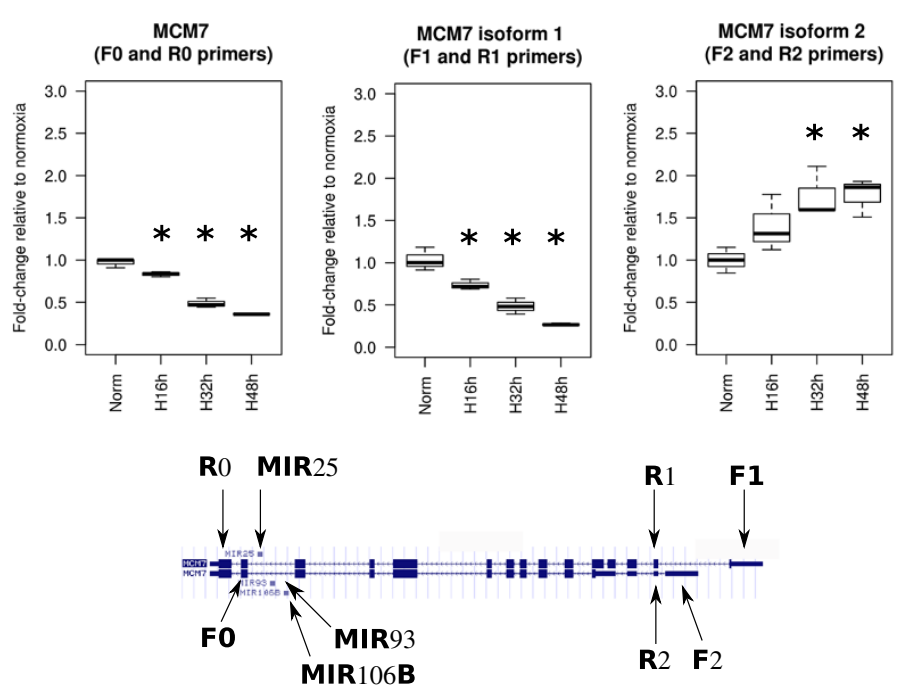

Figure 5 Examples of lack of correlation between microRNA and host gene expression. QPCR was used to assess the expression of two genes hosting microRNAs: FBXW7 (A) and MCM7 (B). Primers able to anneal to different isoforms were used in order to monitor isoform-specific expression. Fold-changes (in linear scale) obtained for each transcript at each hypoxia time point relative to normoxia control are represented in boxplots (*significant fold-change compared to normoxia after ANOVA followed by pairwise t-test, adj.p-val $\leq 0.05$ ). For FBXW7 and MCM7, diagrams showing the different isoforms and the location of microRNAs and pairs of primers designed for detecting each isoform are included in (A) and (B), respectively (forward and reverse primers are designed as $F$ and $R$, respectively).

independent of HIF- $1 \alpha$ or HIF- $2 \alpha$, as their expression did not change in the HIF siRNA experiments (Figure 6).

Despite the fact that some of the changes observed under hypoxia for these genes are HIF dependent, we could not find any high stringency HIF binding site close to any of these genes in our datasets. Since the downregulation of DICER in hypoxic MCF-7 cells is the most strongly associated to HIF among the genes involved in microRNA processing, we decided to test whether this change was also observed in vivo. We used the gene expression data generated for the same breast cancer series we have referred in the previous section (GSE22220) [32] and investigated the correlation of DICER expression with the hypoxia score based on the expression of 99 genes (univariate analysis) [31]. Interestingly, we found that the expression of DICER was significantly inverse correlated to hypoxia in breast cancer (univariate analysis: rho $=-0.37$, pval $=3 . e-08$ ), meaning that the expression of DICER is lower as hypoxia increases. Therefore we show that DICER is also down-regulated under hypoxia in vivo in breast cancer and that this regulation is possibly dependent on HIF although not in a direct manner. 


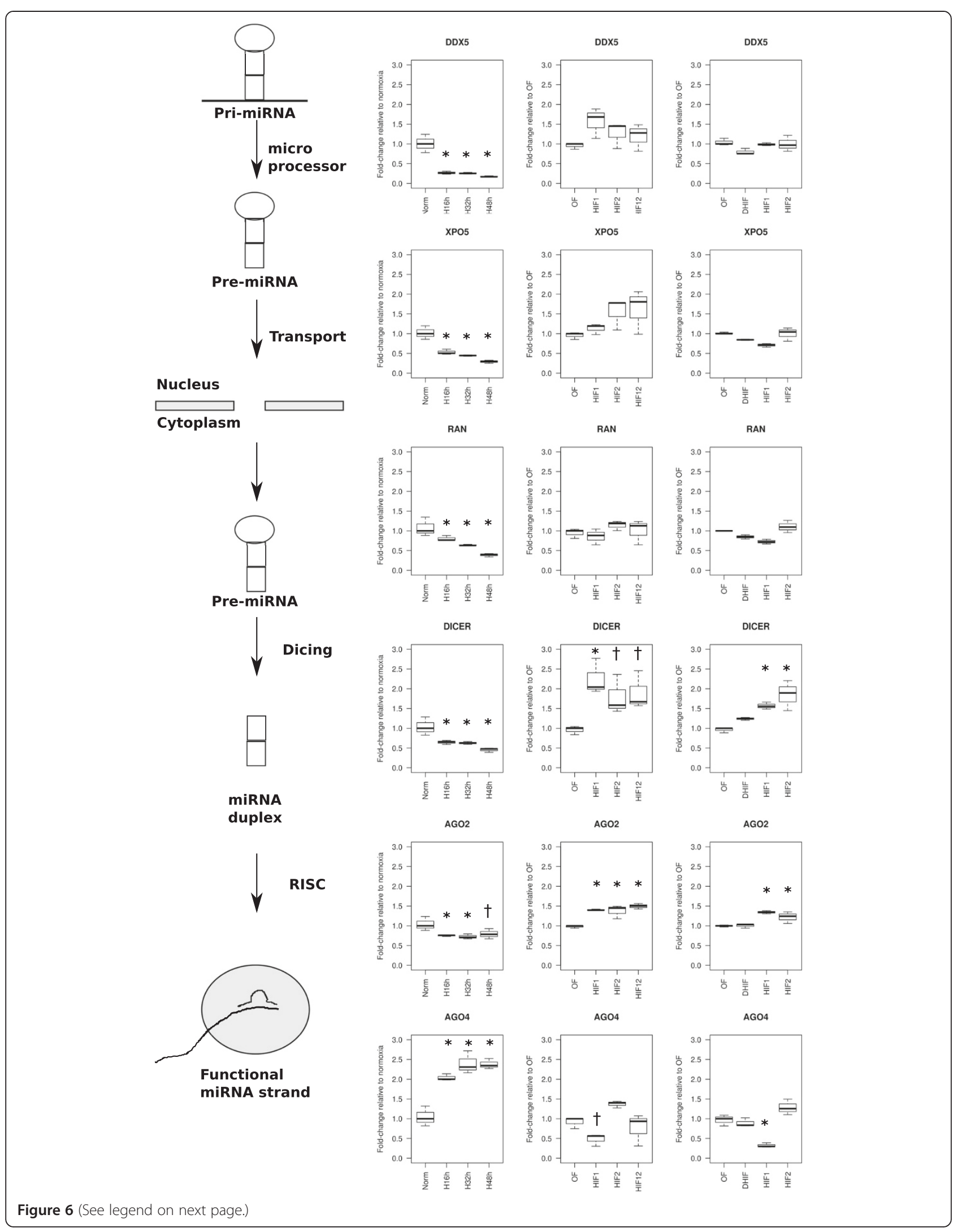




\section{Discussion}

We have presented a very robustly analysed, deep sequencing based microRNA expression profile of a three point hypoxia time course, representing a first for MCF7 cells. To our knowledge, this is the first time such an in-depth study has been performed and all the data is publicly available at GEO (reference series: GSE47534) as a resource for future investigations. We have shown that the number of hypoxia regulated microRNAs increases in time, with a clear breakpoint between $16 \mathrm{~h}$ and $32 \mathrm{~h}$. This is in agreement with Kulshreshtha and colleagues [16], although spotted microarrays provided a limitation of their study. Overall, 69 microRNAs were found regulated in more than one time point, of which 56 are novel for hypoxia. In particular, we report here the down-regulation of hsa-miR-4521, hsa-miR-145-3p and hsa-miR-222-5p at 16 h, $32 \mathrm{~h}$ and $48 \mathrm{~h}$ of hypoxia. These changes were detected along the whole time course in addition to the up-regulation of hsa-miR-210-3p. Therefore, these as yet largely uncharacterised microRNAs could play an important role in hypoxia. Since hsa-miR-210-3p has proved to be a master regulator (for a review see [33]) and an independent prognostic factor in breast cancer [27], it will be worth to assess these microRNAs for their relevance as breast cancer prognostic markers in the future. A top predicted target of hsa-miR-145-3p in miRBase (DIANA MICRO -T and MIRDB) is CCNC (cyclin C), a possible tumor suppressor gene [34], while a top predicted target for hsa-miR4521 is FOXM1 (miRBase, using DIANA MICRO -T and MIRDB), which controls cell cycle gene expression [35]. It is therefore conceivable that the down-regulation of these microRNAs does influence cell proliferation.

In addition, we report other microRNAs that are consistently regulated at $32 \mathrm{~h}$ and $48 \mathrm{~h}$ in MCF-7 cells. Some of these changes have been reported before, using other cell types and conditions, such as the up-regulation of hsa-miR-151-3p in nasopharyngeal carcinoma cells [15], hsa-miR-30d-5p in both nasopharyngeal carcinoma cells and HT29 colon cancer cells [12,15], hsa-miR-140-3p and hsa-miR-143-3p in human primary pulmonary artery smooth muscle cells (PASMCs) [18], hsa-miR-27a-3p and hsa-192-5p in colon and breast cancer cells and PASMCs $[16,18]$, and hsa-miR-1246 and hsa-miR-215-5p in colonic epithelial cells [20]. Also the down-regulation of hsa-miR32-3p and hsa-miR-505-5p has been reported in HUVEC cells [14]. In general, the overlap between studies is limited due to the usage of different cell lines and hypoxia conditions, as well as to the variety of platforms used for the measurement of microRNA expression. It is also not uncommon to find microRNAs for which contrasting regulation under hypoxia has been described. To support this, an extensive comparison of our data at each hypoxia time point with previous studies [11-16,18-20] has been included in Additional file 1: Table S9.

It is known that prolonged hypoxia leads to generation of free radicals and therefore to oxidative stress. Some of the microRNAs found to be regulated by hypoxia in this study have been reported in the context of oxidative stress. For instance, hsa-miR-210-3p regulates mitochondrial activity under hypoxia by targeting ISCU1, ISCU2, COX10, SDHD and NDUFA4 and also affects the production of Reactive Oxygen Species (ROS). Indeed it has been found that hypoxia increases ROS levels in cancer cells and that this effect can be reversed by blocking miR-210-3p. However ROS levels are not altered by hypoxia in endothelial cells but they are increased upon hsa-miR-210-3p abrogation. In other cells such as differentiated skeletal muscle cells, neonatal rat cardiomyocytes and $\mathrm{H} 9 \mathrm{c} 2$ cells, miR-210-3p expression reduces mitochondrial ROS production and decreases mitochondrial mass (all reviewed in [36]). Therefore further work is needed to understand the role of this microRNA in the regulation of ROS levels. It has been also reported that the treatment of auditory cells with the potent oxidant t-BHP down-regulates the expression of hsa-miR-203a and hsa-miR-28-3p (which we found up-regulated in hypoxia at $32 \mathrm{~h}$ and $48 \mathrm{~h}$, Table 1) and hsa-miR-92a-1-5p (which we found down-regulated at $32 \mathrm{~h}$ and $48 \mathrm{~h}$ of hypoxia, Table 2) [37]. Furthermore, the expression of the hypoxia regulated hsa-miR-143-3p, hsa-miR-27a-3p (Table 1) and hsa-miR-22-3p (Table 2) has been described to be altered in human fibroblasts after treatment with peroxide and etoposide (only hsa-miR-27a-3p) [38].

The next question to address was the role of HIF in this process as a key mediator of the transcriptional response to hypoxia. In previous work $[29,39]$, it was demonstrated that HIF- $1 \alpha$ and HIF- $2 \alpha$ binding to DNA is associated with up-regulation of nearby genes, but the association of binding sites and microRNA expression at whole genome level had not been explored so far. Only two microRNAs, hsa-miR-26a and hsa-miR-210, have been shown to have closely positioned HIF binding sites by HIF-1 $\alpha$ chromatin immunoprecipitation (ChIP) [16]. Here, we used HIF- $1 \alpha$ and HIF-2 $\alpha$ ChIP-seq data 
generated from MCF-7 cells to locate HIF binding sites close to microRNAs at genome-wide level. GSEA using up- and down-regulated microRNAs revealed an association between proximity of HIF binding and upregulation. This is further supported when comparing our data to previous studies, since HIF binding sites identified here are closer to microRNAs reported to be up-regulated rather than down-regulated in hypoxia (Additional file 1: Table S9). We showed that functionally important, up-regulated microRNAs have HIF binding sites, such as hsa-miR-27a and hsa-miR-30d. Both microRNAs are involved in cancer progression, hsa-miR-30d-5p by targeting TP53 [40] and hsa-miR-27a-3p by regulating a number of transcription factors such as ZBTB10 $[41,42]$. The HIF dependence of the microRNA cluster composed by hsa-miR-27a, hsa-miR-23a and hsa-miR24-2 had been discussed earlier through the prediction of a conserved HIF binding site within the promoter region of this microRNA cluster [43]. In addition to the HIF binding site, we show here that the expression of hsamiR-27a-3p and hsa-miR-24-3p is positively correlated with a hypoxia score based on the expression of 99 genes in a series of breast cancer samples. This would reinforce the role of HIF in regulating microRNAs in breast cancer and other solid tumours, and the idea that hypoxia could be a key factor in microRNA modulation in cancer [16].

Nevertheless, not all the microRNAs with HIF binding sites in their proximity were up-regulated under hypoxia. We also found some discrepancies between microRNA and host gene expression (where microRNAs are located within intragenic regions), and have shown in the example of the MCM7 locus, that differential expression of transcript isoforms may play a role in these discrepancies. In general, host gene expression was less responsive to hypoxia than changes in levels of mature microRNA forms. This is in agreement with findings that other levels of microRNA regulation take place. Indeed, it has been shown that hypoxia is able to regulate the expression and activity of proteins involved in the post-transcriptional microRNA processing. In HUVEC cells exposed to chronic hypoxia $(24 \mathrm{~h})$, several components of the processing pathway as DGCR8, EXPORTIN5, DICER, TRBP, AGO1 and $\mathrm{AGO} 2$ are significantly down-regulated at both protein and mRNA level [14]. Moreover, the decrease of DICER protein and mRNA levels has been confirmed in vivo in several tissues from mice exposed to chronic hypoxia and also low mRNA levels for DICER have been observed in rat models for pulmonary arterial hypertension [44]. On the other hand, it has been shown in PASMCs that AGO2 can be subjected to a posttranslational modification that increases its stability and translocation rate to the stress granules. Since AGO2 is the key mediator of microRNA function, this modification of AGO2 has an impact on microRNA - target interaction
[18]. Some of our findings are in agreement with these recent reports, such as the down-regulation of XPO5, DICER and AGO2 transcripts. In addition, we found a significant down-regulation of DDX5 and RAN, and a significant increase in AGO4 transcript. We have further explored the regulation of these genes under hypoxia in other 13 cell lines by using expression data available in GEO from other hypoxia studies (see Additional file 1: Table S10). These cell lines were exposed to hypoxia (mostly 1\% Oxygen) for a period ranging between $16 \mathrm{~h}$ and $72 \mathrm{~h}$. In general we can see that DICER, DDX5, XPO5 and $R A N$ are down-regulated at transcriptional level in several cell lines but not all, suggesting that these changes have a component of cell specificity (Additional file 1: Table S10). EIF2C2 (AGO2) transcript presents contrasting regulation depending on the cell line whereas EIF2C4 (AGO4) appears to be up-regulated in two other cell lines (HUVEC and prostate cancer at $48 \mathrm{~h}$ and $72 \mathrm{~h}$ of hypoxia, respectively. Additional file 1: Table S10). Although the transcript levels do not always correspond to protein levels, previous studies in hypoxia (as we have just referred above) show a good concordance between both for several of the microRNA processing genes. DDX5 acts also as a transcription cofactor and is required for cell proliferation [45], for induction of TP53 dependent p21 (CDKN1A) expression after DNA damage [46] and NOTCH1 signalling [47]. The effect of AGO4 on microRNA levels is not clear, but it could provide a further enhancement of RISC complex mediated effects on translation. Despite the fact that several members of the processing machinery are down-regulated, recent work has shown that hydroxylation [18] and phosphorylation [24] of AGO2 affect the microRNA maturation process.

The role of HIF in the regulation of the genes involved in the microRNA processing has not been thoroughly studied. Using HIF siRNA on MCF-7 cells, we showed that indeed DICER and $\mathrm{AGO} 2$ transcriptional downregulations in hypoxia are HIF- $1 \alpha$ and HIF- $2 \alpha$ dependent, while this dependency was not observed in HUVEC cells [14]. Also, AGO4 transcriptional up-regulation in hypoxia is HIF-1 $\alpha$ dependent. Since no HIF binding site was mapped close to these genes in our datasets, the regulation is likely to be indirect and in line with the observation that in breast cancer samples low levels of DICER are associated with a hypoxia signature.

HIF is an important factor in the pathophysiology of cancer and the identification of the mechanisms through which it mediates microRNA expression regulation will lead to a better understanding of this complex pathway. In addition, establishing a complete repertoire of hypoxia regulated microRNAs will lead to more detailed analysis of large scale cancer sample datasets for microRNA-target gene relationships [48] and lead to the development of advanced prognostic assays and therapeutic approaches. 


\section{Conclusions}

Next generation sequencing based methods are state of the art for microRNA profiling. Here we applied this methodology and identified a high number of microRNAs that are regulated by hypoxia in MCF-7 cells, including novel hypoxia regulated microRNAs detected in this study that are candidates for developing breast cancer prognostic assays. The microRNA data were integrated with mRNA and HIF ChIP-seq data to show genome wide correlation between HIF binding and microRNA regulation for the first time. In addition, we show that hsa-miR-27a-3p and hsa-miR-24-3p, for which we have identified a HIF binding site at close distance, are positively correlated to hypoxia in breast cancer. Analysis of microRNA-host gene expression correlation supports the idea that posttranscriptional mechanisms for microRNA expression regulation are in action under hypoxia, and we provide evidence that HIF can play a role by modulating the expression levels of microRNA processing genes such as the down-regulation of DICER. Moreover we report an inverse correlation of DICER expression to hypoxia in breast cancer. Therefore, HIF, as a key factor of the hypoxia transcriptional response plays a role in the regulation of microRNAs either directly through binding to microRNA loci, or indirectly through regulating microRNA processing gene expression and pathways leading to posttranslational modifications of AGO2.

\section{Methods}

\section{Cell culture and hypoxia exposure}

Breast adenocarcinoma cell line MCF-7 cells were grown in Dulbecco's Modified Eagle Medium supplemented with $10 \%$ fetal bovine serum (Sigma-Aldrich, St Loius, Missouri, USA) and $2 \mathrm{mmol} / \mathrm{L}$ of L-glutamine. Exposure of cell cultures to hypoxia (1\% Oxygen) was undertaken in an In vivo2 Hypoxia Work Station (Ruskin Technologies, Pencoed, UK) in parallel with cells maintained in normoxic conditions (21\% oxygen). Cells were recovered after $16 \mathrm{~h}$, $32 \mathrm{~h}$ and $48 \mathrm{~h}$ of exposure to hypoxia. All experiments were done in triplicate from independent cell cultures.

\section{RNA extraction}

RNA was extracted from cells using the miRVana miRNA Isolation Kit (Ambion, Life Technologies, Carlsbad, California, USA). RNA quality and abundance were determined after extraction using an Agilent 2100 Bioanalyzer (Agilent Technologies, Santa Clara, California, USA) and a Nanodrop ND-1000 spectrophotometer (Thermo Fisher Scientific, Waltham, Massachusetts, USA), respectively.

\section{Preparation of microRNA libraries for next-generation sequencing}

For each experimental condition (normoxia, $16 \mathrm{~h}$ hypoxia, $32 \mathrm{~h}$ hypoxia, $48 \mathrm{~h}$ hypoxia), small RNA libraries for next- generation sequencing were prepared from RNA samples obtained from two biological replicates. Each library was prepared from the 20 to 30 nucleotide RNA fraction. This was isolated from $2 \mu \mathrm{g}$ of total RNA after being run in a 15\% urea-TBE gel (Invitrogen, Life technologies, Carlsbad, California, USA) for $1 \mathrm{~h}$ at $200 \mathrm{~V}$. The RNA contained in the excised band was eluted in $300 \mu \mathrm{l}$ of $0.3 \mathrm{M} \mathrm{NaCl}$ by $4 \mathrm{~h}$ of constant rotation at room temperature. The elute was separated from the gel debris using a Spin-X-column (Thermo Fisher Scientific, Waltham, Massachusetts, USA) and RNA was precipitated by adding $750 \mu \mathrm{l}$ of $100 \%$ ethanol and $3 \mu \mathrm{l}$ of glycogen $(1 \mathrm{mg} / \mathrm{ml})$ and incubating for 30 minutes at $-80^{\circ} \mathrm{C}$. The precipitated RNA was centrifuged at $14,000 \mathrm{rpm}$ for 25 minutes at $4^{\circ} \mathrm{C}$, washed with $75 \%$ ethanol, air dried and resuspended in $5 \mu \mathrm{l}$ of RNAsefree water. This material was used to prepare sequencing libraries using the DGE-small RNA sample preparation kit (v1.5) from Illumina (Illumina inc., San Diego, CA, USA ) according to the protocol provided by the manufacturer. The abundance of the libraries was measured using Qubit dsDNA HS Assay kit and a Qubit 2.0 fluorometer (both from Life technologies, Carlsbad, California, USA). The size of the products contained in the libraries was assessed with a DNA 1000 chip and an Agilent 2100 Bioanalyser (both from Agilent Technologies, Santa Clara, California, USA). The libraries were sequenced using an Illumina Hiseq sequencer according to the manufacture's standard protocols at $36 \mathrm{bp}$ read length.

\section{Alignment and analysis of microRNA next-generation sequencing data}

Small RNA sequencing data were processed from raw FASTQ files and analysed using the Kraken pipeline (http://www.ebi.ac.uk/research/enright/software/kraken) [49]. The 3' adaptors were stripped using the criteria: 12-nt alignment stretch with no more than 2 mismatches and no gaps. The reads were then filtered for lowcomplexity regions and the remaining reads were sizeselected for 18-26nt, resulting in inclusion of more than $75 \%$ of reads on average for each sample (Additional file 1: Table S1). A file with unique reads and their corresponding counts was generated for each sample. The final processed unique reads were mapped to the human genome (hg19 based on Ensembl v64 [50]) using Bowtie 0.12.7 [51] allowing for two mismatches and using the best alignment stratum option. Reads mapped to more than 20 loci were discarded. Mapped reads were classified and counted based on genomic annotations (RNAs, coding genes, pseudogenes and repeats) obtained from Ensembl v64 (Additional file 1: Table S1). For reads mapping to multiple loci, counts were divided by total number of reads. The sense and antisense distribution of mapped reads across all chromosomes was also analysed. 
For differential expression of microRNAs, the overlap between mapped reads and human mature microRNAs (based on miRBase v17 [52]) was found using the function findOverlaps available in the Bioconductor GenomicRanges package (http://bioconductor.org/packages/2.6/bioc/html/ GenomicRanges.html). The normalization and differential expression was performed with Bioconductor edgeR package v2.3.57 [53] using both common and tagwise dispersion. The significant differentially expressed microRNAs were determined by an adjusted $\mathrm{p}$ value lower than 0.05 based on the Benjamini and Hochberg multiple testing correction [54]. Data has been submitted to GEO (reference series: GSE47534; miRNA-seq: GSE47602).

\section{HIF-1a and HIF-2a ChIP-seq analysis}

We used the 500 published high-stringency HIF binding sites [29] in order to look for microRNAs that map to within $50 \mathrm{~kb}$ of each binding site. Gene Set Enrichment Analysis (GSEA) was carried out as described previously [29].

\section{microRNA microarrays}

microRNA expression profiles were performed using the human microRNA microarray V2 (Agilent Technologies, Santa Clara, California, USA) which contains 723 human microRNAs (Sanger database V.10.1) and 76 human viral microRNAs. Three biological replicate samples for each experimental condition (normoxia, 16 h hypoxia, $32 \mathrm{~h}$ hypoxia and 48 h hypoxia) were analysed. Samples were prepared with the Mirna Labelling Reagent and Hybridization kit (Agilent Technologies, Santa Clara, California, USA) following the instructions supplied by the manufacturer. The microarray hybridization was performed at $55^{\circ} \mathrm{C}$ for $20 \mathrm{~h}$ and at a constant speed rotation of $20 \mathrm{rpm}$. The hybridization signals were detected by the Agilent Microarray scanner G2565BA and quantified using the Agilent Feature extraction software version 9.5.1 (both from Agilent Technologies, Santa Clara, California, USA). A GeneView file including the Total Gene Signal (raw data) and the Total Gene Error for each of the microRNAs interrogated on the microarray was generated for all the samples and used for further analysis. One sample corresponding to MCF-7 cells exposed to hypoxia for $16 \mathrm{~h}$ was affected by a random technical problem and had to be removed due to the bad quality of the scan.

\section{microRNA microarray data analysis}

The Total Gene Signal was normalised using a variance stabilization method described by Huber and colleagues [55]. Furthermore a detection factor was calculated for each microRNA in every sample as the following subtraction: Total Gene Signal - Total Gene Error. A microRNA was considered detected when the value of this factor was higher than zero. The normalised data was then filtered based on the detection factor and on the microRNA class.
The minimal requirements for a microRNA to be selected for further analysis were: (i) detection in at least two biological replicates of any experimental condition and (ii) be included as human microRNA in miRBase (www.mirbase.org). Limma analysis was then performed to assess independently the effects of $16 \mathrm{~h}, 32 \mathrm{~h}$ and $48 \mathrm{~h}$ of hypoxia exposure compared to normoxic control in microRNA expression levels in MCF-7 cells. Benjamini and Hochberg method was used to correct for multiple testing [54]. microRNAs with adjusted $\mathrm{p}$ values lower than 0.05 were considered significantly regulated. Data has been submitted to GEO (reference series: GSE47534; miRNA: GSE47532).

\section{Gene expression microarrays}

mRNA expression was measured using Illumina HumanWG-6 v3.0 Expression Bead Chip arrays (Illumina inc., San Diego, CA, USA). The Illumina platform was chosen as it performed well in large comparative studies [56]. Three biological replicate samples for each experimental condition (normoxia, $16 \mathrm{~h}$ hypoxia, $32 \mathrm{~h}$ hypoxia and $48 \mathrm{~h}$ hypoxia) were analysed. RNA was amplified using the Illumina Total Prep RNA Amplification Kit (Ambion, Life Technologies, Carlsbad, California, USA). Amplified RNA product (850 ng) was hybridised to the Illumina microarrays using single chamber hybridization cartridges. Washing, staining and scanning were carried out as specified in the Illumina Whole Genome Expression Manual v.1.

\section{Gene expression microarray data analysis}

Average signal was background subtracted with local background subtraction (BeadStudio) and consolidated per gene. Data was quantile normalized in Bioconductor (www.bioconductor.org) and filtered based on gene detection level using the pvalDet parameter. Only genes with pvalDet lower than 0.05 in at least 2 replicates of any of the experimental condition were considered for further analysis. Limma analysis was then performed to assess independently the effects of $16 \mathrm{~h}, 32 \mathrm{~h}$ and $48 \mathrm{~h}$ of hypoxia exposure compared to normoxic control in gene expression levels in MCF-7 cells. Benjamini and Hochberg method was used to correct for multiple testing [54]. Genes with adjusted p values lower than 0.05 were considered significantly regulated. Data has been submitted to GEO (reference series: GSE47534; mRNA: GSE47533).

Datasets from published studies (presented in Additional file 1: Table S10) have been analysed as described above.

\section{Real time PCR (qPCR) analysis}

MicroRNA expression was assessed by qPCR with TaqMan microRNA assay protocol (Applied Biosystems, Life Technologies, Carlsbad, California, USA) using $5 \mathrm{ng}$ of 
total RNA per microRNA as indicated by the manufacturer. QPCR was done in a CFX96 real-time PCR detection system (BioRad, Hercules, California, US). Cycling conditions included a pre-incubation step $\left(95^{\circ} \mathrm{C}\right.$ for 3 minutes) and 40 cycles of amplification $\left(95^{\circ} \mathrm{C}\right.$ for 30 seconds, $60^{\circ} \mathrm{C}$ for 1 minute). For gene expression, total RNA $(2 \mu \mathrm{g})$ was reverse transcribed using the SuperScript II Reverse Transcription kit, 50 ng of random primers (both from Invitrogen, Life technologies, Carlsbad, California, USA) and $0.5 \mathrm{mM}$ dNTP mixture (Bioline, London, UK). QPCR was done in a CFX96 real-time PCR detection system using $30 \mathrm{ng}$ of cDNA, $1 \mathrm{X}$ iQ SYBR-green supermix (Biorad, Hercules, California, US) and primers at a final concentration of $2 \mathrm{mM}$. Cycling conditions included a pre-incubation step $\left(95^{\circ} \mathrm{C}\right.$ for 3 minutes $), 40$ cycles of amplification $\left(95^{\circ} \mathrm{C}\right.$ for 30 seconds, annealing temperature for 30 seconds and $72^{\circ} \mathrm{C}$ for 30 seconds) and a melting curve $\left(95^{\circ} \mathrm{C}\right.$ for 1 minute, $55^{\circ} \mathrm{C}$ for 1 minute, denaturation from $55^{\circ} \mathrm{C}$ to $95^{\circ} \mathrm{C}$ at $0.5^{\circ} \mathrm{C} / 10$ seconds increments). Each reaction was done in triplicate. All primers used are listed in Additional file 1: Table S8.

Expression values were normalised to the geometrical mean of housekeeping genes RPL11, RPL30 and RPS6 and fold-changes between treatments and controls were determined by the $2^{-\Delta \Delta C t}$ method [57] as implemented in the SLqPCR Bioconductor package (http://www.bioconductor.org/packages/2.12/bioc/html/ SLqPCR.html). The variance between sample groups was assessed through the Barlett test for variance. If variances could be assumed as equal between groups, significant differences were established using ANOVA followed by pairwise t-test. If variances could not be assumed as equal, significant differences were then assessed using a one way test for equal means followed by pairwise t-test. After multiple test correction by Benjamini and Hochberg method [54], results were considered significant at two levels: with adjusted $\mathrm{p}$ value lower or equal to 0.05 and with adjusted $\mathrm{p}$ value lower or equal to 0.08. All analysis was done using R v9 (http://www.r-project.org).

\section{Protein immunoblotting}

Cells were lysed in urea/SDS buffer (6.7 M urea, $10 \mathrm{mM}$ Tris-Cl pH 7.4, 10\% glycerol, 1\% SDS) supplemented with $1 \mathrm{mM}$ dithiothreitol. Extracts were resolved by SDS-PAGE, electroblotted onto PVDF membranes (Millipore) and probed with primary antibodies followed by HRP-conjugated secondary antibodies. SuperSignal Chemiluminescent Substrates (Pierce) were used to visualize immunoreactive species. Antibodies used: antiHIF1 $\alpha 610959$ from BD Biosciences, anti-HIF2 $\alpha$ 190b from Peter Ratcliffe Lab, and anti- $\beta$-Actin/HPR ab49900 from Abcam.

\section{Short interfering RNA treatment of MCF-7 cells}

RNA oligonucleotides (Ambion, Life technologies, Carlsbad, California, USA) were used for siRNA suppression of individual $H I F$ family members (HIF-1 $\alpha$ : sense, CUGAU GACCAGCAACUUGAtt; antisense, UCAAGUUGCUG GUCAUCAGtt. HIF-2 $\alpha$; sense, CAGCAUCUUUGAUA GCAGUtt; antisense, ACUGCUAUCAAAGAUGCUGtt). A set of oligonucleotides (Ambion, Life technologies, Carlsbad, California, USA) against a sequence from the Drosophila melanogaster HIF sequence, which lacks any substantial sequence similarity with human HIF or other genes, was used as a negative control (D-HIF: sense, CCUACAUCCCGAUCGAUGAtt; antisense, UCAUCGA UCGGGAUGUAGGtt) in one of the experiments. MCF-7 cells were seeded at $30 \%$ confluence and grown in normoxic conditions. Cells were transfected twice with $20 \mathrm{nmol} / \mathrm{L}$ of siRNA at 24 and 48 hours using OligofectAMINE (Invitrogen, Life technologies, Carlsbad, California, USA) according to the instructions of the manufacturer. At 55 hours, cells were exposed to $1 \%$ oxygen, and after an additional 16 hours, RNA was extracted. Each siRNA treatment was done in triplicate as well as the parallel control using OligofectAMINE reagent alone. The same protocol was used for the generation of protein extracts. We ensured that specific and substantial knockdown was occurring in MCF-7 cells under hypoxia by assaying HIF- $\alpha$ levels by immunoblotting (as previously described [26]).

\section{Statistical methods}

A signature of 99 genes up-regulated under hypoxic conditions has been derived in head and neck tumors in vivo and it has been assessed for its prognostic value in a head and neck and breast cancer data set [31]. Here, this signature was used to calculate a hypoxic score for a breast cancer series using the gene expression profiles (GSE22220) published in [32]. The score is a measure of the level of expression of genes in the hypoxia signature: a high positive score reflects a higher than average expression of the genes in the hypoxia signature and indicates that the sample is hypoxic, a low negative score reflects a lower than average expression of the genes in the hypoxia signature and indicates that the sample is normoxic [31]. Correlation of microRNA expression from the same breast cancer series (GSE22220) and hypoxia score was assessed using Spearman's rank tests. Statistical analyses were done using $\mathrm{R}$.

\section{Additional files}

Additional file 1: Table S1. MicroRNA-seq analysis. Details are given on analysis output at different stages. Table S2. MicroRNAs detected in MCF-7 cells by microRNA-seq. Table S3. MicroRNAs commonly detected by microRNA-seq and Agilent microRNA arrays in MCF-7 cells. Table S4. MicroRNA-seq data for microRNAs within $50 \mathrm{~kb}$ distance of a HIF binding 
site. MicroRNA microarray data also included when available. Table S5. microRNAs encoded within a coding gene sequence. MicroRNA-seq and mRNA microarray expression of corresponding host gene are provided. Table S6. Pairs of microRNA and host genes with low coordination between their expression. Information included as in Table S5. Significant up- and down-regulations are highlighted in red and green, respectively. Table S7. Microarray data for genes involved in the microRNA processing. Table S8. Primers used in the GPCR analysis. Table S9. Comparison with previously published hypoxia studies. MicroRNAs reported to be hypoxia regulated in previous studies are included and the regulation is indicated as up (up-regulated) and down (down-regulated). Concerning our data, microRNAs found significantly regulated at each hypoxia time $(16 \mathrm{~h}, 32 \mathrm{~h}$ and $48 \mathrm{~h}$ ) as well as HIF binding sites identified are included. Hypoxic regulations determined by qPCR are indicated with*. Table S10. Differential expression of microRNA processing genes using data from published hypoxia studies. Significant changes are highlighted in red (for up-regulation) and green (for down-regulation). Note for Table S2 to Table S7 and Table S10: microRNAs or genes (Tables S7 and S10) are arranged in numerical order for ease of reading. Fold change (FC) in linear scale (hypoxia vs normoxia) and p-value before and after multiple test correction (adj.p-val) are shown for each microRNA and gene at each hypoxia time (only adj.p-val is shown in Table S10). Platform specified at the header when multiple data sets are displayed in the same table.

Additional file 2: Figure S1. Validation of microRNA sequencing data by microRNA microarray. RNA samples were hybridised to Agilent microRNA arrays (3 biological replicates per condition). We found 228 microRNAs commonly detected between sequencing and microarray platforms. The fold-changes obtained for each microRNA in each platform have been compared for each hypoxia time and represented in scatter plots in log2 scale. MicroRNAs found significantly regulated by sequencing (adj.p-val <0.05) are highlighted in colours: in blue if they are only significantly regulated in the given hypoxia time point or in red if they are as well significantly regulated in other time points. Solid colours represent microRNAs that were also found significant by microarray analysis (limma, adj.p-val < 0.05) in the particular hypoxia time point. The correlation (pearson) between fold-changes is $0.37(p-v a l=8.47 e-09)$ in hypoxia $16 \mathrm{~h}$, 0.27 (p-val $=2.85 \mathrm{e}-05)$ in hypoxia $32 \mathrm{~h}$ and $0.30(\mathrm{p}-\mathrm{val}=3.75 \mathrm{e}-06)$ in hypoxia $48 \mathrm{~h}$. The correlation is better when only considering the microRNAs found signficantly regulated by sequencing: $0.43(p-v a l=0.0052)$ in hypoxia $32 \mathrm{~h}$ and $0.42(\mathrm{p}-\mathrm{val}=0.0019)$ in hypoxia $48 \mathrm{~h}$.

Additional file 3: Figure S2. Correlation between microRNA expression obtained from microarray data and corresponding host gene expression. Expression of genes hosting microRNAs was obtained from microarray data and expression of corresponding microRNAs was obtained from microRNA microarray data. The hypoxic regulation at $32 \mathrm{~h}$ and $48 \mathrm{~h}$ for both groups was then compared from two different perspectives. First, hosting genes were sorted in 3 groups at each time point: significantly down-regulated (down), not significantly regulated (unv) and significantly up-regulated (up). The fold-change distribution for each group of genes at $32 \mathrm{~h}$ and $48 \mathrm{~h}$ of hypoxia compared to normoxia is shown in boxplots (A). For each group of genes, the fold-change distribution of corresponding microRNAs at $32 \mathrm{~h}$ and $48 \mathrm{~h}$ compared to normoxia is also shown in boxplots for comparison (B). Second, microRNAs hosted within genes were sorted in 3 groups according to their hypoxic regulation at $32 \mathrm{~h}$ and $48 \mathrm{~h}$ : significantly down-regulated (down), not significantly regulated (unv) and significantly up-regulated (up). The fold-change distribution for each group of microRNAs at $32 \mathrm{~h}$ and $48 \mathrm{~h}$ of hypoxia compared to normoxia is shown in boxplots (C). For each group of microRNAs, the fold-change distribution of corresponding host genes at $32 \mathrm{~h}$ and $48 \mathrm{~h}$ compared to normoxia is also shown in boxplots for comparison (D). All fold-change distributions are shown in linear scale.

\section{Abbreviations}

AGO: Argonaute; ChIP: Chromatin immunoprecipitation; ChIP-seq: Chromatin immunoprecipitation sequencing; GSEA: Gene set enrichment analysis; HIF: Hypoxia inducible factor; HUVEC: Human umbilical vein endothelial cells; PASMC: Pulmonary artery smooth muscle cells; Pre-miRNA: Precursor microRNA; Pri-miRNA: Primary microRNA; qPCR: Real time PCR or quantitative PCR; RISC: RNA induced silencing complex; ROS: Reactive oxygen species; siRNA: Short interfering RNA; VHL: Von Hippel Lindau.

\section{Competing interests}

The authors declare that they have no competing interests.

\section{Authors' contributions}

CC did the experimental work and generated the data (microRNA-seq, microRNA and gene expression microarray and QPCR data), participated in the analysis of the different datasets, integrated the results and drafted the manuscript. HKS, MR, JAGA, AGH and AJE participated in the analysis of the microRNA sequencing data. DRM provided the HIF ChIP-seq data and participated in the integration of this data to microRNA-seq data. $\mathrm{HC}$ provided directional ribo-depleted RNA-seq data and participated in the integration of this data to HIF ChIP data. YMT did the HIF protein immunoblots. FMB participated in the analysis of microRNA and gene expression microarray data and the correlations to hypoxia score. ALH participated in the design of the study and helped to draft the manuscript. JR conceived of the study, supervised the experiments and manuscript production. All authors read and approved the final manuscript.

\section{Acknowledgements}

We would like to thank Ernesto Lowy and Richard Mott for initial microRNA sequencing data analysis; Peter Ratcliffe for advise on the manuscript. This work was supported by the Wellcome Trust [075491/Z/04].

\section{Author details}

${ }^{1}$ The Wellcome Trust Centre for Human Genetics, University of Oxford, Roosevelt Drive, Oxford, United Kingdom. ${ }^{2}$ EMBL_European Bioinformatics Institute, Wellcome Trust Genome Campus, Hinxton, United Kingdom. ${ }^{3}$ Henry Wellcome Building for Molecular Physiology, University of Oxford, Roosevelt Drive, Oxford, United Kingdom. ${ }^{4}$ Institute of Molecular Oncology, Biomedical Sciences Research Center "Alexander Fleming", 34 Fleming Street, Vari 16672, Greece. ${ }^{5}$ Cancer Research UK Molecular Oncology Laboratories, Weatherall Institute of Molecular Medicine, University of Oxford, John Radcliffe Hospital, Oxford, United Kingdom. ${ }^{6}$ Department of Biochemistry, Faculty of Science, King Abdulaziz University, Jeddah, Saudi Arabia. ${ }^{7}$ Present Address: McGill University and Genome Quebec Innovation Centre, 740 DR Penfield Ave, Montreal H3A OG1, Canada.

Received: 14 September 2013 Accepted: 5 February 2014 Published: 11 February 2014

\section{References}

1. Harris AL: Hypoxia-a key regulatory factor in tumour growth. Nat Rev Cancer 2002, 2:38-47.

2. Semenza GL: Hypoxia-inducible factor 1: master regulator of $\mathrm{O} 2$ homeostasis. Curr Opin Genet Dev 1998, 8:588-594.

3. Kaelin WG Jr: How oxygen makes its presence felt. Genes Dev 2002, 16:1441-1445.

4. Schofield CJ, Ratcliffe PJ: Oxygen sensing by HIF hydroxylases. Nat Rev Mol Cell Biol 2004, 5:343-354.

5. Griffiths-Jones S, Saini HK, Van Dongen S, Enright AJ: miRBase: tools for microRNA genomics. Nucleic Acids Res 2008, 36:D154-D158.

6. Rodriguez A, Griffiths-Jones S, Ashurst JL, Bradley A: Identification of mammalian microRNA host genes and transcription units. Genome Res 2004, 14:1902-1910.

7. Lee Y, Jeon K, Lee JT, Kim S, Kim VN: MicroRNA maturation: stepwise processing and subcellular localization. EMBO J 2002, 21:4663-4670.

8. Treiber T, Treiber N, Meister $\mathrm{G}$ : Regulation of microRNA biogenesis and function. Thromb Haemost 2012, 107:605-610.

9. Okamura K, Hagen JW, Duan H, Tyler DM, Lai EC: The mirtron pathway generates microRNA-class regulatory RNAs in Drosophila. Cell 2007, 130:89-100

10. Packer AN, Xing Y, Harper SQ, Jones L, Davidson BL: The bifunctional microRNA miR-9/miR-9* regulates REST and CoREST and is downregulated in Huntington's disease. J Neurosci 2008, 28:14341-14346.

11. Donker RB, Mouillet JF, Nelson DM, Sadovsky Y: The expression of Argonaute 2 and related microRNA biogenesis proteins in normal and hypoxic trophoblasts. Mol Hum Reprod 2007, 13:273-279.

12. Guimbellot JS, Erickson SW, Mehta T, Wen H, Page GP, Sorscher EJ, Hong JS: Correlation of microRNA levels during hypoxia with predicted target mRNAs through genome-wide microarray analysis. BMC Med Genomics 2009, 2:15. 
13. Hebert C, Norris K, Scheper MA, Nikitakis N, Sauk JJ: High mobility group A2 is a target for miRNA-98 in head and neck squamous cell carcinoma. Mol Cancer 2007, 6:5

14. Ho JJ, Metcalf JL, Yan MS, Turgeon PJ, Wang JJ, Chalsev M, Petruzziello-Pellegrini TN, Tsui AK, He JZ, Dhamko H, et al: Functional importance of dicer protein in the adaptive cellular response to hypoxia. J Biol Chem 2012, 287:29003-29020.

15. Hua Z, Lv Q, Ye W, Wong CK, Cai G, Gu D, Ji Y, Zhao C, Wang J, Yang BB, Zhang Y: MiRNA-directed regulation of VEGF and other angiogenic factors under hypoxia. PLoS One 2006, 1:e116.

16. Kulshreshtha R, Ferracin M, Wojcik SE, Garzon R, Alder H, Agosto-Perez FJ, Davuluri R, Liu CG, Croce CM, Negrini M, et al: A microRNA signature of hypoxia. Mol Cell Biol 2007, 27:1859-1867.

17. Voellenkle C, Rooij J, Guffanti A, Brini E, Fasanaro P, Isaia E, Croft L, David M, Capogrossi MC, Moles A, et al: Deep-sequencing of endothelial cells exposed to hypoxia reveals the complexity of known and novel microRNAs. RNA 2012, 18:472-484.

18. Wu C, So J, Davis-Dusenbery BN, Qi HH, Bloch DB, Shi Y, Lagna G, Hata A: Hypoxia potentiates microRNA-mediated gene silencing through posttranslational modification of Argonaute2. Mol Cell Biol 2011, 31:4760-4774

19. Pulkkinen K, Malm T, Turunen M, Koistinaho J, Yla-Herttuala S: Hypoxia induces microRNA miR-210 in vitro and in vivo ephrin-A3 and neuronal pentraxin 1 are potentially regulated by miR-210. FEBS Lett 2008, 582:2397-2401.

20. Bruning U, Cerone L, Neufeld Z, Fitzpatrick SF, Cheong A, Scholz CC, Simpson DA, Leonard MO, Tambuwala MM, Cummins EP, Taylor CT: MicroRNA-155 promotes resolution of hypoxia-inducible factor 1alpha activity during prolonged hypoxia. Mol Cell Biol 2011, 31:4087-4096.

21. Crosby ME, Kulshreshtha R, Ivan M, Glazer PM: MicroRNA regulation of DNA repair gene expression in hypoxic stress. Cancer Res 2009, 69:1221-1229.

22. Lei Z, Li B, Yang Z, Fang H, Zhang GM, Feng ZH, Huang B: Regulation of HIF-1alpha and VEGF by miR-20b tunes tumor cells to adapt to the alteration of oxygen concentration. PLoS One 2009, 4:e7629.

23. Loayza-Puch F, Yoshida Y, Matsuzaki T, Takahashi C, Kitayama H, Noda M: Hypoxia and RAS-signaling pathways converge on, and cooperatively downregulate, the RECK tumor-suppressor protein through microRNAs. Oncogene 2010, 29:2638-2648.

24. Shen J, Xia W, Khotskaya YB, Huo L, Nakanishi K, Lim SO, Du Y, Wang Y, Chang WC, Chen $\mathrm{CH}$, et al: EGFR modulates microRNA maturation in response to hypoxia through phosphorylation of AGO2. Nature 2013, 497:383-387.

25. Appelhoff RJ, Tian YM, Raval RR, Turley H, Harris AL, Pugh CW, Ratcliffe PJ, Gleadle JM: Differential function of the prolyl hydroxylases PHD1, PHD2, and PHD3 in the regulation of hypoxia-inducible factor. J Biol Chem 2004, 279:38458-38465

26. Elvidge GP, Glenny L, Appelhoff RJ, Ratcliffe PJ, Ragoussis J, Gleadle JM: Concordant regulation of gene expression by hypoxia and 2-oxoglutarate-dependent dioxygenase inhibition: the role of HIF-1alpha, HIF-2alpha, and other pathways. J Biol Chem 2006, 281:15215-15226.

27. Camps C, Buffa FM, Colella S, Moore J, Sotiriou C, Sheldon H, Harris AL, Gleadle JM, Ragoussis J: hsa-miR-210 Is induced by hypoxia and is an independent prognostic factor in breast cancer. Clin Cancer Res 2008, 14:1340-1348.

28. Pritchard CC, Cheng HH, Tewari M: MicroRNA profiling: approaches and considerations. Nat Rev Genet 2012, 13:358-369.

29. Schodel J, Oikonomopoulos S, Ragoussis J, Pugh CW, Ratcliffe PJ, Mole DR: High-resolution genome-wide mapping of HIF-binding sites by ChIP-seq. Blood 2011, 117:e207-e217.

30. Choudhry H, Schodel J, Oikonomopoulos S, Camps C, Grampp S, Harris AL, Ratcliffe PJ, Ragoussis J, Mole DR: Extensive regulation of the non-coding transcriptome by hypoxia: role of HIF in releasing paused RNApol2. EMBO Rep 2014, 15:70-76.

31. Winter SC, Buffa FM, Silva P, Miller C, Valentine HR, Turley H, Shah KA, Cox GJ, Corbridge RJ, Homer JJ, et al: Relation of a hypoxia metagene derived from head and neck cancer to prognosis of multiple cancers. Cancer Res 2007, 67:3441-3449.

32. Buffa FM, Camps C, Winchester L, Snell CE, Gee HE, Sheldon H, Taylor M, Harris AL, Ragoussis J: microRNA-associated progression pathways and potential therapeutic targets identified by integrated mRNA and microRNA expression profiling in breast cancer. Cancer Res 2011, 71:5635-5645.
33. Chan SY, Loscalzo J: MicroRNA-210: a unique and pleiotropic hypoxamir. Cell Cycle 2010, 9:1072-1083.

34. Ohata N, Ito S, Yoshida A, Kunisada T, Numoto K, Jitsumori Y, Kanzaki H, Ozaki T, Shimizu K, Ouchida M: Highly frequent allelic loss of chromosome 6q16-23 in osteosarcoma: involvement of cyclin C in osteosarcoma. Int J Mol Med 2006, 18:1153-1158.

35. Chen X, Muller GA, Quaas M, Fischer M, Han N, Stutchbury B, Sharrocks AD, Engeland $\mathrm{K}$ : The forkhead transcription factor FOXM1 controls cell cycle-dependent gene expression through an atypical chromatin binding mechanism. Mol Cell Biol 2013, 33:227-236.

36. Magenta A, Greco S, Gaetano C, Martelli F: Oxidative stress and microRNAs in vascular diseases. Int J Mol Sci 2013, 14:17319-17346.

37. Wang Z, Liu Y, Han N, Chen X, Yu W, Zhang W, Zou F: Profiles of oxidative stress-related microRNA and mRNA expression in auditory cells. Brain Res 2010, 1346:14-25

38. Simone NL, Soule BP, Ly D, Saleh AD, Savage JE, Degraff W, Cook J, Harris CC, Gius D, Mitchell JB: lonizing radiation-induced oxidative stress alters miRNA expression. PLoS One 2009, 4:e6377.

39. Mole DR, Blancher C, Copley RR, Pollard PJ, Gleadle JM, Ragoussis J, Ratcliffe PJ: Genome-wide association of hypoxia-inducible factor (HIF)-1alpha and HIF-2alpha DNA binding with expression profiling of hypoxia-inducible transcripts. J Biol Chem 2009, 284:16767-16775.

40. Kumar M, Lu Z, Takwi AA, Chen W, Callander NS, Ramos KS, Young KH, Li Y: Negative regulation of the tumor suppressor p53 gene by microRNAs. Oncogene 2011, 30:843-853.

41. Li X, Mertens-Talcott SU, Zhang S, Kim K, Ball J, Safe S: MicroRNA-27a indirectly regulates estrogen receptor $\{$ alpha\} expression and hormone responsiveness in MCF-7 breast cancer cells. Endocrinology 2010, 151:2462-2473.

42. Mertens-Talcott SU, Chintharlapalli S, Li X, Safe S: The oncogenic microRNA-27a targets genes that regulate specificity protein transcription factors and the G2-M checkpoint in MDA-MB-231 breast cancer cells. Cancer Res 2007, 67:11001-11011.

43. Kulshreshtha R, Ferracin M, Negrini M, Calin GA, Davuluri RV, Ivan M: Regulation of microRNA expression: the hypoxic component. Cell Cycle 2007, 6:1426-1431.

44. Caruso P, MacLean MR, Khanin R, McClure J, Soon E, Southgate M, MacDonald RA, Greig JA, Robertson KE, Masson R, et al: Dynamic changes in lung microRNA profiles during the development of pulmonary hypertension due to chronic hypoxia and monocrotaline. Arterioscler Thromb Vasc Biol 2010, 30:716-723.

45. Mazurek A, Luo W, Krasnitz A, Hicks J, Powers RS, Stillman B: DDX5 regulates DNA replication and is required for cell proliferation in a subset of breast cancer cells. Cancer Discov 2012, 2:812-825.

46. Nicol SM, Bray SE, Derek Black H, Lorimore SA, Wright EG, Lane DP Meek DW, Coates PJ, Fuller-Pace FV: The RNA helicase p68 (DDX5) is selectively required for the induction of p53-dependent p21 expression and cell-cycle arrest after DNA damage. Oncogene 2013, 32:3461-3469.

47. Lin S, Tian L, Shen H, Gu Y, Li JL, Chen Z, Sun X, James You M, Wu L: DDX5 is a positive regulator of oncogenic NOTCH1 signaling in T cell acute lymphoblastic leukemia. Oncogene 2013, 32:4845-4853.

48. Dvinge H, Git A, Graf S, Salmon-Divon M, Curtis C, Sottoriva A, Zhao Y, Hirst M, Armisen J, Miska EA, et al: The shaping and functional consequences of the microRNA landscape in breast cancer. Nature 2013, 497:378-382.

49. Davis MP, Van Dongen S, Abreu-Goodger C, Bartonicek N, Enright AJ: Kraken: a set of tools for quality control and analysis of high-throughput sequence data. Methods 2013, 63:41-49.

50. Flicek P, Amode MR, Barrell D, Beal K, Brent S, Carvalho-Silva D, Clapham P, Coates G, Fairley S, Fitzgerald S, et al: Ensembl. Nucleic Acids Res 2012, 40:D84-D90.

51. Langmead B, Trapnell C, Pop M, Salzberg SL: Ultrafast and memory-efficient alignment of short DNA sequences to the human genome. Genome Biol 2009, 10:R25.

52. Kozomara A, Griffiths-Jones S: miRBase: integrating microRNA annotation and deep-sequencing data. Nucleic Acids Res 2011, 39:D152-D157.

53. Robinson MD, McCarthy DJ, Smyth GK: edgeR: a bioconductor package for differential expression analysis of digital gene expression data. Bioinformatics 2010, 26:139-140.

54. Benjamini $Y$, Hochberg $Y$ : Controlling the false discovery rate - a practical and powerful approach to multiple testing. J Royal Stat Soc SeriesB-Methodol 1995, 57:289-300. 
55. Huber W, von Heydebreck A, Sueltmann H, Poustka A, Vingron M: Parameter estimation for the calibration and variance stabilization of microarray data. Stat Appl Genet Mol Biol 2003, 2. Article3.

56. Shi L, Reid LH, Jones WD, Shippy R, Warrington JA, Baker SC, Collins PJ, de Longueville F, Kawasaki ES, Lee KY, et al: The MicroArray Quality Control (MAQC) project shows inter- and intraplatform reproducibility of gene expression measurements. Nat Biotechnol 2006, 24:1151-1161.

57. Livak KJ, Schmittgen TD: Analysis of relative gene expression data using real-time quantitative PCR and the 2(-Delta Delta $C(T))$ method. Methods 2001, 25:402-408.

doi:10.1186/1476-4598-13-28

Cite this article as: Camps et al:: Integrated analysis of microRNA and mRNA expression and association with HIF binding reveals the complexity of microRNA expression regulation under hypoxia. Molecular Cancer 2014 13:28.

\section{Submit your next manuscript to BioMed Central and take full advantage of:}

- Convenient online submission

- Thorough peer review

- No space constraints or color figure charges

- Immediate publication on acceptance

- Inclusion in PubMed, CAS, Scopus and Google Scholar

- Research which is freely available for redistribution 